\title{
COMPLETE ASYMPTOTIC EXPANSIONS FOR EIGENVALUES OF DIRICHLET LAPLACIAN IN THIN THREE-DIMENSIONAL RODS*
}

\author{
Denis Borisov ${ }^{1}$ and Giuseppe Cardone ${ }^{2}$
}

\begin{abstract}
We consider the Dirichlet Laplacian in a thin curved three-dimensional rod. The rod is finite. Its cross-section is constant and small, and rotates along the reference curve in an arbitrary way. We find a two-parametric set of the eigenvalues of such operator and construct their complete asymptotic expansions. We show that this two-parametric set contains any prescribed number of the first eigenvalues of the considered operator. We obtain the complete asymptotic expansions for the eigenfunctions associated with these first eigenvalues.
\end{abstract}

Mathematics Subject Classification. 35P05, 35J05, 35B25, 35C20.

Received October 20, 2009. Revised February 22, 2010 and April 6, 2010.

Published online August 6, 2010.

\section{INTRODUCTION}

The asymptotics of the spectra of elliptic operators in thin domains were studied by many authors, see, for instance $[2-10,12,14]$, and the references therein. There are two types of thin domains usually considered, namely, thin rods and thin plates. Both types were considered in the book [12]. The eigenvalues for elliptic operators with the Neumann boundary condition on the lateral surface of the rods and on the bases of the plates were studied. The asymptotic expansions for the eigenvalues and the eigenfunctions were constructed and justified. We also mention the survey [9] on thin rods.

A thin two-dimensional domain formed by two different thin rectangles was studied in [14], i.e., the boundary of the domain was non-smooth. The operator considered was the Laplacian subject to the Neumann condition on the bases and to Dirichlet one on the lateral boundary. The paper provided the asymptotic expansions for the eigenvalues remaining bounded as the width of the domain tends to zero, and the asymptotics for the associated eigenfunctions. These asymptotic expansions were rigorously justified. The Laplacian in a thin two-dimensional domain was also considered in [8]. The domain had a variable width with the unique point of maximum. The uniform resolvent convergence was established and two-terms asymptotics for the eigenvalues were obtained, as well as convergence theorems for the associated eigenfunctions. In [7] these results were extended for an infinite

Keywords and phrases. Thin rod, Dirichlet Laplacian, eigenvalue, asymptotics.

* D.B. was partially supported by RFBR (10-01-00118), by the grant of the President of Russia for young scientists-doctors of sciences (MD-453.2010.1) and for Leading Scientific School (NSh-6249.2010.1), by Federal Task Program (contract 02.740.11. 0612), and by the grant of FCT (ptdc/mat/101007/2008). The work was partially supported by the project "Progetto ISA: Attivita' di Internazionalizzazione dell Universita' degli Studi del Sannio".

1 Bashkir State Pedagogical University, October Revolution St. 3a, 450000 Ufa, Russia. borisovdi@yandex.ru

2 University of Sannio, Department of Engineering, Corso Garibaldi, 107, 82100 Benevento, Italy. gcardone@unisannio.it 
thin strip under similar conditions for the width. We also mention the paper [10], where a thin strip (finite or infinite) was considered with the Neumann condition on the upper boundary and with the Dirichlet condition on the lower boundary. Here two-terms asymptotics for the first eigenvalues were constructed. The case of a curved infinite strip was also studied in [5], where the number of the discrete eigenvalues below the essential spectrum was estimated. The results of [8] were also extended in [2]. Here a two-parametric set of the eigenvalues was found and their complete asymptotic expansions were constructed.

A finite three-dimensional rod was considered in [3]. The cross section was supposed to be constant and to rotate along the reference curve in an arbitrary way. Two-terms asymptotics for the first eigenvalues were constructed and convergence theorems for the associated eigenfunctions were established. Similar results were obtained in [6] for a tube in a space of arbitrary dimension. An infinite three-dimensional thin tube with a round cross section was studied in [5]. The number of the discrete eigenvalues below the essential spectrum was estimated and their complete asymptotic expansions were constructed. We also mention the paper [4], where a multi-dimensional thin cylinder with distorted ends was considered. The operator studied was the Laplacian in such domain subject to the Dirichlet condition on the lateral surface and to the Neumann one on the distorted ends. The attention was paid to the localization effect of some eigenfunctions at the distorted ends. The asymptotics for these eigenfunctions and the corresponding eigenvalues were constructed.

In this paper we extend the results of [3]. We again consider the Dirichlet Laplacian in a curved thin rod. The cross section of the rod is a fixed domain, which rotates along the reference curve in an arbitrary way. In what follows this operator, its eigenvalues and eigenfunctions are referred to as the perturbed ones. We find a two-parametric set of the perturbed eigenvalues and construct their complete asymptotic expansions. The eigenvalues are indexed by the first two terms of their asymptotic expansions. Namely, the leading terms are determined by the eigenvalues of the Dirichlet Laplacian on the cross-section of the rod. Each of the leading terms determines a certain operator on the reference curve, and its eigenvalues are the next-to-leading terms of the aforementioned asymptotic expansions for the perturbed eigenvalues. It is convenient to group the perturbed eigenvalues into a countable set of series, where each series consists of the perturbed eigenvalues with the same leading term in the asymptotic expansions. We show that the series associated with the smallest leading term contains any prescribed number of the first eigenvalues of the perturbed operator provided the rod is thin enough. We prove that these eigenvalues are simple and construct complete asymptotic expansions for the associated eigenfunctions.

In conclusion to this section, we describe briefly the contents of the paper. The next section contains the description of the problem and the main results. In the third section we introduce a change of variables required for the constructing the asymptotic expansions. In the fourth section we select the aforementioned two-parametric series of the eigenvalues and construct their asymptotic expansions. In the last fifth section we describe the first eigenvalues of the perturbed operator and give the asymptotic expansions for the associated eigenfunctions.

\section{Formulation of the Problem And the main RESUlts}

Let $x=\left(x_{1}, x_{2}, x_{3}\right)$ be Cartesian coordinates in $\mathbb{R}^{3}, \gamma$ be a finite infinitely differentiable curve in $\mathbb{R}^{3}$ without self-intersections. By $s$ and $s_{0}$ we denote the arc length and the length of $\gamma, s \in\left[0, s_{0}\right]$. We parameterize $\gamma$ by its arc length, and $\boldsymbol{r}=\boldsymbol{r}(s)$ is the infinitely differentiable vector describing $\gamma$. The tangential vector of $\gamma$ is indicated by $\boldsymbol{\tau}=\boldsymbol{\tau}(s)$. By $\boldsymbol{\eta}=\boldsymbol{\eta}(s)$ we denote an infinitely differentiable in $s \in\left[0, s_{0}\right]$ unit vector defined on $\gamma$ being orthogonal to $\boldsymbol{\tau}(s)$ for all $s \in\left[0, s_{0}\right]$. We let $\boldsymbol{\beta}(s):=\boldsymbol{\tau}(s) \times \boldsymbol{\eta}(s)$, where $\times$ is the cross product. It is clear that $\boldsymbol{\beta}(s)$ is infinitely differentiable in $s \in\left[0, s_{0}\right]$, and $(\boldsymbol{\tau}, \boldsymbol{\eta}, \boldsymbol{\beta})$ is an orthonormalized frame on $\gamma$. One of the possible choices of $\boldsymbol{\eta}$ is

$$
\boldsymbol{\eta}(s):=\cos \alpha(s) \boldsymbol{n}(s)+\sin \alpha(s) \boldsymbol{b}(s),
$$

where $\boldsymbol{n}=\boldsymbol{n}(s)$ and $\boldsymbol{b}=\boldsymbol{b}(s)$ are the normal and binormal vectors of $\gamma$, and $\alpha(s) \in C^{\infty}\left[0, s_{0}\right]$ is an arbitrary function describing how our frame rotates with respect to the Frenet one. It follows from (1.1) that

$$
\boldsymbol{\beta}(s):=-\sin \alpha(s) \boldsymbol{n}(s)+\cos \alpha(s) \boldsymbol{b}(s) .
$$


Although this formula and (1.1) could be an appropriate definition of $\boldsymbol{\eta}$ and $\boldsymbol{\beta}$, we do not use this way. The reason is that the Frenet frame does not exists for all smooth curves, since the normal vector can be undefined at the points, where $\boldsymbol{r}^{\prime \prime}(s)=0$.

By $\omega$ we indicate a bounded domain in $\mathbb{R}^{2}$ with an infinitely smooth boundary, and the symbol $\varepsilon$ stands for a small positive parameter. We introduce a thin curved rod as

$$
\Omega_{\varepsilon}:=\left\{x \in \mathbb{R}^{3}: x=\boldsymbol{r}(s)+\varepsilon \xi_{2} \boldsymbol{\eta}(s)+\varepsilon \xi_{3} \boldsymbol{\beta}(s), s \in\left(0, s_{0}\right),\left(\xi_{2}, \xi_{3}\right) \in \omega\right\} .
$$

Since the curve $\gamma$ is smooth and not self-intersecting, the $\operatorname{rod} \Omega_{\varepsilon}$ has no self-intersections for $\varepsilon$ small enough. Hereinafter the parameter $\varepsilon$ is assumed to be chosen exactly in such way.

The main object of our study is the spectrum of the Dirichlet Laplacian in $L_{2}\left(\Omega_{\varepsilon}\right)$, and this operator is denoted by $\mathcal{H}_{\varepsilon}$. We introduce this operator rigorously as the Friedrichs extension of $-\Delta_{x}$ on $C_{0}^{\infty}\left(\Omega_{\varepsilon}\right)$. For each $\varepsilon>0$ the operator $\mathcal{H}_{\varepsilon}$ has a compact resolvent and its spectrum is thus purely discrete. The aim of this paper is to construct the complete asymptotic expansions for the eigenvalues of $\mathcal{H}_{\varepsilon}$. We also observe that the eigenvalues of $\mathcal{H}_{\varepsilon}$ can be equivalently regarded as those of the boundary value problem

$$
-\Delta \psi(\cdot, \varepsilon)=\lambda(\varepsilon) \psi(\cdot, \varepsilon) \quad \text { in } \Omega_{\varepsilon}, \quad \psi(\cdot, \varepsilon)=0 \quad \text { on } \partial \Omega_{\varepsilon}, \quad \psi(\cdot, \varepsilon) \in W_{2}^{1}\left(\Omega_{\varepsilon}\right) .
$$

In order to formulate the main results we need to introduce additional notations. Let $W_{2,0}^{2}(\omega)$ be the subspace of $W_{2}^{2}(\omega)$ consisting of the functions vanishing on $\partial \omega$. In the same way we introduce the space $W_{2,0}^{2}\left(0, s_{0}\right)$. By $\mathcal{S}$ we indicate the Dirichlet Laplacian in $L_{2}(\omega)$ with $W_{2,0}^{2}(\omega)$ as the domain. This operator is self-adjoint. Let $\lambda_{n}$ be the eigenvalues of $\mathcal{S}$ arranged in the ascending order with the multiplicities taken into account,

$$
\lambda_{1}<\lambda_{2} \leqslant \lambda_{3} \leqslant \ldots \leqslant \lambda_{n} \leqslant \ldots
$$

By $\phi_{n}$ we denote the associated eigenfunctions orthonormalized in $L_{2}(\omega)$. By the smoothness improving theorems [11], Chapter IV, Section 2.3, the functions $\phi_{n}$ are infinitely differentiable in $\bar{\omega}$.

It is straightforward to check that

$$
\boldsymbol{\tau}^{\prime}=\kappa_{1} \boldsymbol{\eta}-\kappa_{2} \boldsymbol{\beta}, \quad \boldsymbol{\eta}^{\prime}=-\kappa_{1} \boldsymbol{\tau}+\kappa_{3} \boldsymbol{\beta}, \quad \boldsymbol{\beta}^{\prime}=\kappa_{2} \boldsymbol{\tau}-\kappa_{3} \boldsymbol{\eta}
$$

where $\kappa_{i}=\kappa_{i}(s) \in C^{\infty}\left[0, s_{0}\right]$ are certain functions characterizing the geometric properties of $\gamma$ and rotation of $\boldsymbol{\eta}$.

Let an eigenvalue $\lambda_{n}$ be simple. Denote

$$
\mathcal{R}:=\xi_{3} \frac{\partial}{\partial \xi_{2}}-\xi_{2} \frac{\partial}{\partial \xi_{3}}, \quad C_{n}(\omega):=\int_{\omega}\left|\mathcal{R} \phi_{n}\right|^{2} \mathrm{~d} \xi .
$$

By $\mathcal{L}_{n}$ we indicate the operator

$$
-\frac{\mathrm{d}^{2}}{\mathrm{~d} s^{2}}+C_{n}(\omega) \kappa_{3}^{2}(s)-\frac{\kappa_{1}^{2}(s)+\kappa_{2}^{2}(s)}{4}
$$

in $L_{2}\left(0, s_{0}\right)$ with the domain $W_{2,0}^{2}\left(0, s_{0}\right)$. The operator $\mathcal{L}_{n}$ is self-adjoint. Since it is one-dimensional, by Cauchy theorem we conclude that all its eigenvalues are simple. We indicate these eigenvalues by $\lambda_{0}^{(n, m)}, m=1,2, \ldots$ and arrange them in the ascending order. Let $\Psi_{0}^{(n, m)}$ be the associated eigenfunctions orthonormalized in $L_{2}\left(0, s_{0}\right)$.

If one chooses $\boldsymbol{\eta}$ and $\boldsymbol{\beta}$ in accordance with (1.1), (1.2), it yields

$$
\kappa_{1}=\kappa \cos \alpha, \quad \kappa_{2}=\kappa \sin \alpha, \quad \kappa_{3}=\alpha^{\prime}+\varkappa,
$$


where $\kappa=\kappa(s)$ and $\varkappa=\varkappa(s)$ are the curvature and torsion of $\gamma$, and $\kappa, \varkappa \in C^{\infty}\left[0, s_{0}\right]$. In this case the operator $\mathcal{L}_{n}$ becomes

$$
-\frac{\mathrm{d}^{2}}{\mathrm{~d} s^{2}}+C_{n}(\omega)\left(\alpha^{\prime}(s)+\varkappa(s)\right)^{2}-\frac{\kappa^{2}(s)}{4} .
$$

Our first result gives the complete asymptotic expansions for the eigenvalues of $\mathcal{H}_{\varepsilon}$.

Theorem 1.1. Let $\varepsilon$ be small enough. Assume $\lambda_{n}$ is a simple eigenvalue of $\mathcal{S}$. Then there exists a twoparametric set of the eigenvalues $\lambda^{(n, m)}(\varepsilon)$ of $\mathcal{H}_{\varepsilon}$ with the asymptotics

$$
\lambda^{(n, m)}(\varepsilon)=\varepsilon^{-2} \lambda_{n}+\lambda_{0}^{(n, m)}+\sum_{i=1}^{K} \varepsilon^{i} \lambda_{i}^{(n, m)}+\mathcal{O}\left(\varepsilon^{K+1}\right)
$$

for any $K \geqslant 1$, where

$$
\begin{aligned}
& \lambda_{1}^{(n, m)}=\left(\psi_{0}^{(n, m)}, Q^{(n, m)} \psi_{0}^{(n, m)}\right)_{L_{2}(\Omega)}+2\left(\mathcal{R} \psi_{0}^{(n, m)}, \kappa_{3}^{2} \mathrm{q} \mathcal{R} \psi_{0}^{(n, m)}\right)_{L_{2}(\Omega)}, \\
& Q^{(n, m)}:=\left(2 \lambda_{0}^{(n, m)}-\left(2 C_{n}(\omega)-\frac{1}{2}\right) \kappa_{3}^{2}\right) \mathrm{q}+\frac{1}{2} \frac{\partial^{2} \mathrm{q}}{\partial s^{2}}+\frac{1}{2} \kappa_{3}^{\prime} \mathcal{R} \mathrm{q}, \\
& \mathrm{q}=\mathrm{q}(s, \xi):=\kappa_{1}(s) \xi_{2}-\kappa_{2}(s) \xi_{3}, \quad \psi_{0}^{(n, m)}=\psi_{0}^{(n, m)}(s, \xi):=\Psi_{0}^{(n, m)}(s) \phi_{n}(\xi) .
\end{aligned}
$$

The remaining coefficients in the asymptotic series (1.5) are given by the formulas (3.44) in Lemma 3.1.

We observe that the set of the eigenvalues described in Theorem 1.1 is two-parametric and is indexed by $n$ and $m$. Given $n$, the eigenvalues $\lambda^{(n, m)}(\varepsilon)$ form a series with the same leading term. We stress that Theorem 1.1 does not imply that the eigenvalues $\lambda^{(n, m)}(\varepsilon)$ form the whole set of the eigenvalues of $\mathcal{H}_{\varepsilon}$. The first reason is the assumption on the simplicity of $\lambda_{n}$. And even without this assumption it is an additional problem to find out whether the eigenvalues $\lambda^{(n, m)}(\varepsilon)$ are the only possible ones or not.

We make the assumption that $\lambda_{n}$ is simple in order to simplify the calculations in the formal constructing of the asymptotic expansion, see Section 3. If $\lambda_{n}$ is multiple, it is also possible to construct the asymptotic expansions, but the formal constructing becomes more complicated and requires some additional careful calculations. Another interesting issue is the multiplicities of the perturbed eigenvalues corresponding to a multiple eigenvalue $\lambda_{n}$. In view of these issues we regard the case of multiple eigenvalue $\lambda_{n}$ as an additional problem, which we postpone for another article.

One more interesting question is on the asymptotic expansions for the eigenfunctions associated with $\lambda^{(n, m)}(\varepsilon)$. As usually, to justify such asymptotic expansions, one has to know lower bounds for the distances between the perturbed eigenvalues. The structure of the eigenvalues $\lambda^{(n, m)}(\varepsilon)$ is such that it is rather difficult to obtain such bounds once the eigenvalues are bigger than $\varepsilon^{-2} \lambda_{2}$. If we consider only the first eigenvalues of $\mathcal{H}_{\varepsilon}$ lying between $\varepsilon^{-2} \lambda_{1}$ and $\varepsilon^{-2} \lambda_{2}$, it is possible to prove the mentioned lower bounds and to obtain the asymptotic expansions for the associated eigenfunctions. This is our second main result. Before formulating it, we introduce two additional notations,

$$
\Omega:=\left\{(s, \xi): 0<s<s_{0}, \xi \in \omega\right\}, \quad \Omega^{(t)}:=\left\{(s, \xi): t<s<s_{0}-t, \xi \in \omega\right\}, \quad t \in\left(0, s_{0} / 2\right) .
$$

Theorem 1.2. Given any $M \geqslant 1$, there exists $\varepsilon_{0}=\varepsilon_{0}(M)>0$ such that for all $\varepsilon<\varepsilon_{0}$ the first $M$ eigenvalues of $\mathcal{H}_{\varepsilon}$ are $\lambda^{(1, m)}(\varepsilon), m=1, \ldots, M$, satisfying (1.5). These eigenvalues are simple and the associated eigenfunctions have the asymptotics

$$
\psi^{(1, m)}(x(s, \varepsilon \xi), \varepsilon)=\Psi_{0}^{(1, m)}(s) \phi_{1}(\xi)+\sum_{i=1}^{K} \varepsilon^{i} \psi_{i}^{(1, m)}(s, \xi)+\mathcal{O}\left(\varepsilon^{K+1}\right)
$$


for any $K \geqslant 1$, where the coefficients of the series are given by (3.41) in Lemma 3.1. The asymptotics hold true in $W_{2}^{1}(\Omega)$-norm and $C^{k}\left(\overline{\Omega^{(t)}}\right)$-norms for all $k \geqslant 0, t \in\left(0, s_{0} / 2\right)$.

The results of [3] consist of the two-term asymptotics for $\lambda^{(1, m)}(\varepsilon)$ and the leading term in the asymptotics for the eigenfunctions $\psi^{(1, m)}$. The asymptotics for the eigenfunctions were obtained in $L_{2}(\Omega)$. Theorem 1.2 extends these results in two directions. First, it gives the complete asymptotic expansions. Second, the asymptotics of the eigenfunctions are given in a stronger norm. One more extension is provided by Theorem 1.1. Namely, in addition to the first series of the eigenvalues $\lambda^{(1, m)}(\varepsilon)$ described in [3], we provide a countable set of similar series $\lambda^{(n, m)}(\varepsilon), n \geqslant 2$.

One more difference from [3] is the technique employed. The study in [3] was based on $\Gamma$-convergence of certain functionals. Our approach consists of two main steps. The first step is the formal constructing of the asymptotic expansions for the eigenvalues and the eigenfunctions by the multiscale method [1]. The second step is the estimating of error terms by a result from spectral perturbation theory, see [15], Lemmas 12 and 13 . We mention that the same approach based on the formal constructing and the results from the spectral perturbation theory has already been used successfully in studying thin domains, see for instance [14].

\section{Change of variables}

In this section we transform the operator $\mathcal{H}_{\varepsilon}$ to another one which will be more convenient in proving Theorems 1.1 and 1.2 .

Let $y=\left(y_{2}, y_{3}\right)$ be Cartesian coordinates in the plane spanned over $\boldsymbol{\eta}$ and $\boldsymbol{\beta}$ with the axes along these vectors so that the variable $y_{2}$ corresponds to $\boldsymbol{\eta}$ and the variable $y_{3}$ does to $\boldsymbol{\beta}$. We first pass to the variables $(s, y)$ and the domain $\Omega_{\varepsilon}$ is mapped onto

$$
\widetilde{\Omega}_{\varepsilon}:=\left\{(s, y): 0<s<s_{0}, \varepsilon^{-1} y \in \omega\right\} .
$$

At the second step we rescale the variables $y$ passing to variables $(s, \xi)$, where $\xi=\left(\xi_{2}, \xi_{3}\right)=\varepsilon^{-1} y$. Then the domain $\widetilde{\Omega}_{\varepsilon}$ is mapped onto $\Omega$.

We define the operator describing the passing to the variables $(s, y)$ as

$$
(\mathcal{U} u)(s, y)=u(x(s, y)), \quad\left(\mathcal{U}^{-1} u\right)(x)=u(s(x), y(x)) .
$$

We let

$$
\widetilde{\mathcal{H}}_{\varepsilon}:=\mathrm{p} \mathcal{U} \mathcal{H}_{\varepsilon} \mathcal{U}^{-1}, \quad \mathrm{p}=\mathrm{p}(s, y):=1-\mathrm{q}(s, y), \quad \mathrm{q}(s, y)=\kappa_{1}(s) y_{2}-\kappa_{2}(s) y_{3} .
$$

If $\lambda(\varepsilon)$ and $\psi(\cdot, \varepsilon)$ are an eigenvalue and an associated eigenfunction of $\mathcal{H}_{\varepsilon}$, it is clear that the function $\mathcal{U} \psi(\cdot, \varepsilon)$ solves the equations

$$
\mathcal{U H}_{\varepsilon} \mathcal{U}^{-1} \mathcal{U} \psi(\cdot, \varepsilon)=\lambda(\varepsilon) \mathcal{U} \psi_{\varepsilon}, \quad \widetilde{\mathcal{H}}_{\varepsilon} \mathcal{U} \psi(\cdot, \varepsilon)=\lambda(\varepsilon) \mathrm{p} \mathcal{U} \psi(\cdot, \varepsilon) .
$$

Let us obtain the differential expression for $\widetilde{\mathcal{H}}_{\varepsilon}$. Taking into account (1.3) and differentiating the identity

$$
x=\boldsymbol{r}(s)+y_{2} \boldsymbol{\eta}(s)+y_{3} \boldsymbol{\beta}(s)
$$

with respect to $s, y_{2}$, and $y_{3}$, we obtain

$$
\frac{\partial x}{\partial s}=\mathrm{p} \boldsymbol{\tau}(s)-\kappa_{3} y_{3} \boldsymbol{\eta}(s)+\kappa_{3} y_{2} \boldsymbol{\beta}(s), \quad \frac{\partial x}{\partial y_{2}}=\boldsymbol{\eta}(s), \quad \frac{\partial x}{\partial y_{3}}=\boldsymbol{\beta}(s) .
$$

Thus, the derivatives with respect to $x$ and $(s, y)$ are related by the identity

$$
\nabla_{(s, y)}=\mathrm{P} \nabla_{x}
$$


where $\mathrm{P}$ is the matrix with the rows

$$
\mathrm{P}:=\left(\begin{array}{c}
\mathrm{p} \boldsymbol{\tau}-\kappa_{3} y_{3} \boldsymbol{\eta}+\kappa_{3} y_{2} \boldsymbol{\beta} \\
\boldsymbol{\eta} \\
\boldsymbol{\beta}
\end{array}\right)
$$

It is easy to check that

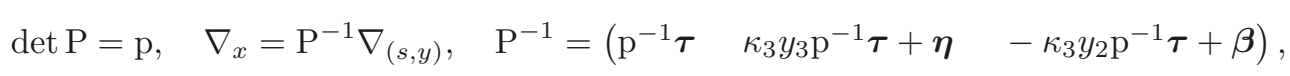

where the vectors in the definition of $\mathrm{P}^{-1}$ are treated as columns. Since $\varepsilon^{-1} y \in \omega$, we have $y=\mathcal{O}(\varepsilon)$ and hence $\mathrm{q}(s, y)=\mathcal{O}(\varepsilon)$. It yields that the function $\mathrm{p}(s, y)$ is strictly positive for the considered values of $y$.

By (2.2) for each $u_{1}, u_{2} \in C_{0}^{\infty}\left(\widetilde{\Omega}_{\varepsilon}\right)$ we have

$$
\begin{aligned}
\left(\widetilde{\mathcal{H}}_{\varepsilon} u_{1}, u_{2}\right)_{L_{2}\left(\widetilde{\Omega}_{\varepsilon}\right)} & =\left(\mathrm{p} \mathcal{U} \mathcal{H}_{\varepsilon} \mathcal{U}^{-1} u_{1}, u_{2}\right)_{L_{2}\left(\widetilde{\Omega}_{\varepsilon}\right)}=\left(\mathcal{H}_{\varepsilon} \mathcal{U}^{-1} u_{1}, \mathcal{U}^{-1} u_{2}\right)_{L_{2}\left(\Omega_{\varepsilon}\right)}=\left(\nabla_{x} \mathcal{U}^{-1} u_{1}, \nabla_{x} \mathcal{U}^{-1} u_{2}\right)_{L_{2}\left(\Omega_{\varepsilon}\right)} \\
& =\left(\mathrm{P}^{-1} \nabla_{(s, y)} u_{1}, \mathrm{p} \mathrm{P}^{-1} \nabla_{(s, y)} u_{2}\right)_{L_{2}\left(\widetilde{\Omega}_{\varepsilon}\right)}=-\left(\operatorname{div}_{(s, y)} \mathrm{p}\left(\mathrm{P}^{-1}\right)^{t} \mathrm{P}^{-1} \nabla_{(s, y)} u_{1}, u_{2}\right)_{L_{2}\left(\widetilde{\Omega}_{\varepsilon}\right)}
\end{aligned}
$$

and therefore

$$
\begin{gathered}
\widetilde{\mathcal{H}}_{\varepsilon}:=-\operatorname{div}(s, y) \\
\mathrm{A}=\left(A_{i j}\right)_{i, j=\overline{1,3}}=\left(\begin{array}{ccc}
\mathrm{p}_{(s, y)} & \kappa_{3} y_{3} \mathrm{p}^{-1} & -\kappa_{3} y_{2} \mathrm{p}^{-1} \\
\kappa_{3} y_{3} \mathrm{p}^{-1} & \mathrm{p}+\kappa_{3}^{2} y_{3}^{2} \mathrm{p}^{-1} & -\kappa_{3}^{2} y_{2} y_{3} \mathrm{p}^{-1} \\
-\kappa_{3} y_{2} \mathrm{p}^{-1} & -\kappa_{3}^{2} y_{2} y_{3} \mathrm{p}^{-1} & \mathrm{p}+\kappa_{3}^{2} y_{2}^{2} \mathrm{p}^{-1}
\end{array}\right) .
\end{gathered}
$$

Now we pass to the variables $\xi$. It leads us to a final transformed operator

$$
\begin{gathered}
\widehat{\mathcal{H}}_{\varepsilon}=-\left(\frac{\partial}{\partial s} A_{11}^{(\varepsilon)} \frac{\partial}{\partial s}+\varepsilon^{-1} \sum_{i=2}^{3} \frac{\partial}{\partial \xi_{i}} A_{i 1}^{(\varepsilon)} \frac{\partial}{\partial s}+\varepsilon^{-1} \sum_{i=2}^{3} \frac{\partial}{\partial s} A_{1 i}^{(\varepsilon)} \frac{\partial}{\partial \xi_{i}}+\varepsilon^{-2} \sum_{i, j=2}^{3} \frac{\partial}{\partial \xi_{i}} A_{i j}^{(\varepsilon)} \frac{\partial}{\partial \xi_{j}}\right) \\
\mathrm{A}^{(\varepsilon)}:=\left(A_{i j}^{(\varepsilon)}\right)_{i, j=\overline{1,3}}=\left(\begin{array}{ccc}
\mathrm{p}_{\varepsilon}^{-1} & \varepsilon \kappa_{3} \xi_{3} \mathrm{p}_{\varepsilon}^{-1} & -\varepsilon \kappa_{3} \xi_{2} \mathrm{p}_{\varepsilon}^{-1} \\
\varepsilon \kappa_{3} \xi_{3} \mathrm{p}_{\varepsilon}^{-1} & \mathrm{p}_{\varepsilon}+\varepsilon^{2} \kappa_{3}^{2} \xi_{3}^{2} \mathrm{p}_{\varepsilon}^{-1} & -\varepsilon^{2} \kappa_{3}^{2} \xi_{2} \xi_{3} \mathrm{p}_{\varepsilon}^{-1} \\
-\varepsilon \kappa_{3} \xi_{2} \mathrm{p}_{\varepsilon}^{-1} & -\varepsilon^{2} \kappa_{3}^{2} \xi_{2} \xi_{3} \mathrm{p}_{\varepsilon}^{-1} & \mathrm{p}_{\varepsilon}+\varepsilon^{2} \kappa_{3}^{2} \xi_{2}^{2} \mathrm{p}_{\varepsilon}^{-1}
\end{array}\right),
\end{gathered}
$$

where

$$
\mathrm{p}_{\varepsilon}(s, \xi):=1-\varepsilon \mathrm{q}(s, \xi),
$$

and the operator $\widehat{\mathcal{H}}_{\varepsilon}$ is considered in $L_{2}(\Omega)$ as the Friedrichs extension from $C_{0}^{\infty}(\Omega)$. The eigenvalue equation (2.1) casts into the form

$$
\widehat{\mathcal{H}}_{\varepsilon} \psi(\cdot, \varepsilon)=\lambda(\varepsilon) \mathrm{p}_{\varepsilon} \psi(\cdot, \varepsilon)
$$

where we redenoted $(\mathcal{U} \psi)(s, \varepsilon \xi, \varepsilon)$ by $\psi(s, \xi, \varepsilon)$.

\section{Proof of Theorem 1.1}

In this section we prove Theorem 1.1. The proof is divided into two parts, the first being devoted to the formal constructing of the asymptotic expansions. The second part consists in proving the existence of the mentioned two-parametric set of the eigenvalues and in the justification of the formal asymptotic expansions for these eigenvalues, i.e., establishing estimates for the error terms. 
We construct the asymptotic expansions for the eigenvalues and the associated eigenfunctions as the series

$$
\begin{aligned}
& \lambda^{(n, m)}(\varepsilon)=\sum_{i=-2}^{\infty} \varepsilon^{i} \lambda_{i}^{(n, m)}, \\
& \psi^{(n, m)}(x, \varepsilon)=\sum_{i=0}^{\infty} \varepsilon^{i} \psi_{i}^{(n, m)}(s, \xi) .
\end{aligned}
$$

The aim of the formal constructing is to determine the coefficients of these series.

We expand the functions $A_{i j}^{(\varepsilon)}$ in the powers of $\varepsilon$,

$$
\begin{array}{ll}
A_{i j}^{(\varepsilon)}=\sum_{k=0}^{\infty} \varepsilon^{k} A_{k}^{(i j)}, \quad A_{k}^{(i j)}=A_{k}^{(j i)}, & \\
A_{k}^{(11)}=\mathrm{q}^{k}, & k \geqslant 0, \\
A_{0}^{(12)}=0, \quad A_{k}^{(12)}=\kappa_{3} \xi_{3} \mathrm{q}^{k-1}, & k \geqslant 1, \\
A_{0}^{(13)}=0, \quad A_{k}^{(13)}=-\kappa_{3} \xi_{2} \mathrm{q}^{k-1}, & k \geqslant 1, \\
A_{0}^{(22)}=1, \quad A_{1}^{(22)}=-\mathrm{q}, \quad A_{k}^{(22)}=\kappa_{3}^{2} \xi_{3}^{2} \mathrm{q}^{k-2}, & k \geqslant 2, \\
A_{0}^{(23)}=A_{1}^{(23)}=0, \quad A_{k}^{(23)}=-\kappa_{3}^{2} \xi_{2} \xi_{3} \mathrm{q}^{k-2}, & k \geqslant 2, \\
A_{0}^{(33)}=1, \quad A_{1}^{(33)}=-\mathrm{q}, \quad A_{k}^{(33)}=\kappa_{3}^{2} \xi_{2}^{2} \mathrm{q}^{k-2}, & k \geqslant 2 .
\end{array}
$$

Hereinafter $\mathrm{q}=\mathrm{q}(s, \xi)$, if else is not specified.

We substitute (2.4), (3.1), (3.2), (3.3), (3.4) into (2.7) and equate the coefficients at the same powers of $\varepsilon$. Calculating the coefficient at $\varepsilon^{i-2}, i \geqslant 0$, we obtain

$$
\begin{aligned}
& \left(-\Delta_{\xi}-\lambda_{-2}^{(n, m)}\right) \psi_{i}^{(n, m)}=\sum_{j=1}^{i} \lambda_{j-2}^{(n, m)} \psi_{i-j}^{(n, m)}+F_{i}^{(n, m)} \quad \text { in } \Omega, \quad \psi_{i}^{(n, m)}=0 \quad \text { on } \partial \Omega, \\
& F_{i}^{(n, m)}:=\sum_{j=1}^{i}\left(\mathcal{F}_{j}-\lambda_{j-3}^{(n, m)} \mathrm{q}\right) \psi_{i-j}^{(n, m)}, \\
& \mathcal{F}_{1}:=-\frac{\partial}{\partial \xi_{2}} \mathrm{q} \frac{\partial}{\partial \xi_{2}}-\frac{\partial}{\partial \xi_{3}} \mathrm{q} \frac{\partial}{\partial \xi_{3}}, \\
& \mathcal{F}_{j}:=\frac{\partial}{\partial s} A_{j-2}^{(11)} \frac{\partial}{\partial s}+\sum_{l=2}^{3} \frac{\partial}{\partial \xi_{l}} A_{j-1}^{(l 1)} \frac{\partial}{\partial s}+\sum_{l=2}^{3} \frac{\partial}{\partial s} A_{j-1}^{(1 l)} \frac{\partial}{\partial \xi_{l}}+\sum_{t, l=2}^{3} \frac{\partial}{\partial \xi_{t}} A_{j}^{(t l)} \frac{\partial}{\partial \xi_{l}} \\
& =\frac{\partial}{\partial s} \mathrm{q}^{j-2} \frac{\partial}{\partial s}+\mathcal{R} \kappa_{3} \mathrm{q}^{j-2} \frac{\partial}{\partial s}+\frac{\partial}{\partial s} \kappa_{3} \mathrm{q}^{j-2} \mathcal{R}+\kappa_{3}^{2} \mathcal{R} \mathrm{q}^{j-2} \mathcal{R}, \quad j \geqslant 2 .
\end{aligned}
$$

Consider problem (3.5) for $i=0$. It is clear that its solution can be chosen as

$$
\psi_{0}^{(n, m)}(s, \xi)=\Psi_{0}^{(n, m)}(s) \phi_{n}(\xi), \quad \lambda_{-2}^{(n, m)}=\lambda_{n},
$$

where the function $\Psi_{0}^{(n, m)}$ is unknown and should satisfy the boundary conditions

$$
\Psi_{0}^{(n, m)}(0)=\Psi_{0}^{(n, m)}\left(s_{0}\right)=0
$$


The eigenvalue equation for $\phi_{n}$

$$
\left(\Delta_{\xi}+\lambda_{n}\right) \phi_{n}=0 \quad \text { in } \omega
$$

and the definition of $\mathcal{F}_{1}$ and q imply

$$
\left(\mathcal{F}_{1}-\lambda_{n} \mathrm{q}\right) \phi_{n}=-\left(\kappa_{1} \frac{\partial}{\partial \xi_{2}}-\kappa_{2} \frac{\partial}{\partial \xi_{3}}\right) \phi_{n} .
$$

Taking into account this formula and (3.8), we write problem (3.5) for $i=1$,

$$
\begin{aligned}
\left(-\Delta_{\xi}-\lambda_{n}\right) \psi_{1}^{(n, m)} & =-\Psi_{0}^{(n, m)}\left(\kappa_{1} \frac{\partial}{\partial \xi_{2}}-\kappa_{2} \frac{\partial}{\partial \xi_{3}}\right) \phi_{n}+\lambda_{-1}^{(n, m)} \Psi_{0}^{(n, m)} \phi_{n} \quad \text { in } \Omega \\
\psi_{1}^{(n, m)} & =0 \quad \text { on } \partial \Omega .
\end{aligned}
$$

Employing equation (3.10), by direct calculations we check that

$$
\left(-\Delta_{\xi}-\lambda_{n}\right) \mathrm{q} \phi_{n}=-2\left(\kappa_{1} \frac{\partial}{\partial \xi_{2}}-\kappa_{2} \frac{\partial}{\partial \xi_{3}}\right) \phi_{n} .
$$

Hence, the problem (3.12) is solvable for

with a solution given by the identity

$$
\lambda_{-1}^{(n, m)}=0
$$

$$
\psi_{1}^{(n, m)}(s, \xi)=\frac{1}{2} \Psi_{0}^{(n, m)}(s) \phi_{n}(\xi) \mathrm{q}(s, \xi)+\Psi_{1}^{(n, m)}(s) \phi_{n}(\xi),
$$

where the function $\Psi_{1}^{(n, m)}$ is unknown and should satisfy the boundary conditions

$$
\Psi_{1}^{(n, m)}(0)=\Psi_{1}^{(n, m)}\left(s_{0}\right)=0 .
$$

The formula (3.14) for $\lambda_{-1}^{(n, m)}$ and that for $\lambda_{0}^{(n, m)}$ in (3.8) prove the formulas for the first terms in (1.5).

We substitute the formulas (3.6), (3.7), (3.8), (3.11), (3.14) into problem (3.5) for $i=2$ to obtain

$$
\begin{gathered}
\left(-\Delta_{\xi}-\lambda_{n}\right) \psi_{2}^{(n, m)}=\lambda_{0}^{(n, m)} \psi_{0}^{(n, m)}+F_{2}^{(n, m)} \quad \text { in } \Omega, \quad \psi_{2}^{(n, m)}=0 \quad \text { on } \partial \Omega, \\
F_{2}^{(n, m)}=\frac{1}{2}\left(\mathcal{F}_{1}-\lambda_{n} \mathrm{q}\right) \mathrm{q} \psi_{0}^{(n, m)}+\mathcal{F}_{2} \psi_{0}^{(n, m)}-\Psi_{1}^{(n, m)}\left(\kappa_{1} \frac{\partial}{\partial \xi_{2}}-\kappa_{2} \frac{\partial}{\partial \xi_{3}}\right) \phi_{n} .
\end{gathered}
$$

Using equation (3.10), by direct calculations we check

$$
\left(\mathcal{F}_{1}-\lambda_{n} \mathrm{q}\right) \mathrm{q} \phi_{n}=-3 \mathrm{q}\left(\kappa_{1} \frac{\partial}{\partial \xi_{2}}-\kappa_{2} \frac{\partial}{\partial \xi_{3}}\right) \phi_{n}-\left(\kappa_{1}^{2}+\kappa_{2}^{2}\right) \phi_{n} .
$$

Hence, we can rewrite the formula for $F_{2}^{(n, m)}$ as follows,

$$
\begin{aligned}
F_{2}^{(n, m)} & =\widetilde{\mathcal{F}} \psi_{0}^{(n, m)}-\Psi_{1}^{(n, m)}\left(\kappa_{1} \frac{\partial}{\partial \xi_{2}}-\kappa_{2} \frac{\partial}{\partial \xi_{3}}\right) \phi_{n}, \\
\widetilde{\mathcal{F}} \psi_{0}^{(n, m)} & =\frac{1}{2}\left(\mathcal{F}_{1}-\lambda_{n} \mathrm{q}\right) \mathrm{q} \psi_{0}^{(n, m)}+\mathcal{F}_{2} \psi_{0}^{(n, m)}, \\
\widetilde{\mathcal{F}}: & =-\frac{3 \mathrm{q}}{2}\left(\kappa_{1} \frac{\partial}{\partial \xi_{2}}-\kappa_{2} \frac{\partial}{\partial \xi_{3}}\right)-\frac{\kappa_{1}^{2}+\kappa_{2}^{2}}{2}+\frac{\partial^{2}}{\partial s^{2}}+\left(\kappa_{3} \frac{\partial}{\partial s}+\frac{\partial}{\partial s} \kappa_{3}\right) \mathcal{R}+\kappa_{3}^{2} \mathcal{R}^{2} .
\end{aligned}
$$


In (3.16) the Laplace operator is taken only with respect to $\xi$, and this problem involves $s$ as a parameter. So, we can consider (3.16) as a problem for the Dirichlet Laplacian $\mathcal{S}$ in $\omega$ with a dependence on $s$. Since $\lambda_{n}$ is a simple eigenvalue of $\mathcal{S}$, the solvability condition of (3.16) is the orthogonality of the right hand side in the equation to $\phi_{n}$ in $L_{2}(\omega)$,

$$
\lambda_{0}^{(n, m)} \Psi_{0}^{(n, m)}+\left(F_{2}^{(n, m)}, \phi_{n}\right)_{L_{2}(\omega)}=0, \quad s \in\left(0, s_{0}\right),
$$

where we have taken into account the normalization of $\phi_{n}$ and the formula (3.8). Let us evaluate the second term in the left hand side of (3.22).

Integrating by parts, we obtain

$$
\begin{aligned}
\int_{\omega} \phi_{n} \mathrm{q}\left(\kappa_{1} \frac{\partial}{\partial \xi_{2}}-\kappa_{2} \frac{\partial}{\partial \xi_{3}}\right) \phi_{n} \mathrm{~d} \xi & =\frac{1}{2} \int_{\omega} \mathrm{q}\left(\kappa_{1} \frac{\partial}{\partial \xi_{2}}-\kappa_{2} \frac{\partial}{\partial \xi_{3}}\right) \phi_{n}^{2} \mathrm{~d} \xi \\
& =-\frac{1}{2} \int_{\omega} \phi_{n}^{2}\left(\kappa_{1} \frac{\partial}{\partial \xi_{2}}-\kappa_{2} \frac{\partial}{\partial \xi_{3}}\right) \mathrm{q} \mathrm{d} \xi=-\frac{\kappa_{1}^{2}+\kappa_{2}^{2}}{2}, \\
\int_{\omega} \phi_{n} \mathcal{R} \phi_{n} \mathrm{~d} \xi & =\frac{1}{2} \int_{\omega} \mathcal{R} \phi_{n}^{2} \mathrm{~d} \xi=0 \\
\int_{\omega} \phi_{n} \mathcal{R}^{2} \phi_{n} \mathrm{~d} \xi & =-\int_{\omega}\left|\mathcal{R} \phi_{n}\right|^{2} \mathrm{~d} \xi=-C_{n}(\omega) \\
\int_{\omega} \phi_{n}\left(\kappa_{1} \frac{\partial}{\partial \xi_{2}}-\kappa_{2} \frac{\partial}{\partial \xi_{3}}\right) \phi_{n} \mathrm{~d} \xi & =\frac{1}{2} \int_{\omega}\left(\kappa_{1} \frac{\partial}{\partial \xi_{2}}-\kappa_{2} \frac{\partial}{\partial \xi_{3}}\right) \phi_{n}^{2} \mathrm{~d} \xi=0
\end{aligned}
$$

We substitute the identities obtained, (3.8), (3.19), (3.21) into (3.22) and arrive at the equation

$$
\frac{\partial^{2} \Psi_{0}^{(n, m)}}{\partial s^{2}}+\left(\frac{\kappa_{1}^{2}+\kappa_{2}^{2}}{4}-\kappa_{3}^{2} C_{n}(\omega)\right) \Psi_{0}^{(n, m)}+\lambda_{0}^{(n, m)} \Psi_{0}^{(n, m)}=0, \quad s \in\left(0, s_{0}\right)
$$

Together with the boundary condition (3.9) it can be rewritten as

$$
\mathcal{L}_{n} \Psi_{0}^{(n, m)}=\lambda_{0}^{(n, m)} \Psi_{0}^{(n, m)} .
$$

This equation is in fact the solvability condition of (3.16), and we satisfy this condition by choosing appropriate $\lambda_{0}^{(n, m)}$ and $\Psi_{0}^{(n, m)}$. Namely, we choose them to be a (simple) eigenvalue and the associated eigenfunction of $\mathcal{L}_{n}$. In what follows the eigenfunctions $\Psi_{0}^{(n, m)}$ are assumed to be orthonormalized in $L_{2}\left(0, s_{0}\right)$. We also note that by the smoothness improving theorems $\Psi_{0}^{(n, m)} \in C^{\infty}\left[0, s_{0}\right]$.

Let $V_{n}$ be the orthogonal complement to $\left\{\phi_{n}\right\}$ in $L_{2}(\omega)$. By $\mathcal{S}_{n}^{\perp}$ we denote the restriction of $\mathcal{S}$ on $V_{n} \cap W_{2,0}^{2}(\omega)$. It is clear that the operator $\left(\mathcal{S}_{n}^{\perp}-\lambda_{n}\right)^{-1}$ is well-defined in and bounded as that from $V_{n}$ in $W_{2,0}^{2}(\omega)$.

It follows from (3.23) that

$$
\left(\kappa_{1} \frac{\partial}{\partial \xi_{2}}-\kappa_{2} \frac{\partial}{\partial \xi_{3}}\right) \phi_{n} \in V_{n}
$$

The identity (3.22) is satisfied due to (3.24) and it yields $\lambda_{0}^{(n, m)} \psi_{0}^{(n, m)}+F_{2}^{(n, m)} \in V_{n}$. Hence, by (3.19), (3.25) we have $\left(\widetilde{\mathcal{F}}+\lambda_{0}^{(n, m)}\right) \psi_{0}^{(n, m)} \in V_{n}$. Taking into account this fact, (3.19), and (3.13), we return back to problem (3.16), 
and write its solution as

$$
\begin{aligned}
\psi_{2}^{(n, m)}(s, \xi) & =\widetilde{\psi}_{2}^{(n, m)}(s, \xi)+\frac{1}{2} \Psi_{1}^{(n, m)}(s) \phi_{n}(\xi) \mathrm{q}(s, \xi)+\Psi_{2}^{(n, m)}(s) \phi_{n}(\xi), \\
\widetilde{\psi}_{2}^{(n, m)}: & =\left(\mathcal{S}_{n}^{\perp}-\lambda_{n}\right)^{-1}\left(\widetilde{\mathcal{F}}+\lambda_{0}^{(n, m)}\right) \psi_{0}^{(n, m)}
\end{aligned}
$$

where the function $\Psi_{2}^{(n, m)}$ is unknown and should satisfy the boundary conditions

$$
\Psi_{2}^{(n, m)}(0)=\Psi_{2}^{(n, m)}\left(s_{0}\right)=0
$$

Bearing in mind the belongings $\phi_{n} \in C^{\infty}(\bar{\omega}), \kappa_{i} \in C^{\infty}\left[0, s_{0}\right]$, and the identities (3.21), (1.4), it is easy to see that the function $\left(\widetilde{\mathcal{F}}+\lambda_{0}^{(n, m)}\right) \psi_{0}^{(n, m)}$ admits separation of variables and can be represented as a finite sum of the terms $\Psi(s) F(\xi)$, where $\Psi \in C^{\infty}\left[0, s_{0}\right]$, and $F \in C^{\infty}(\bar{\omega}) \cap V_{n}$. Thus, the function $\widetilde{\psi}_{2}^{(n, m)}$ is also a finite sum of the terms $\Psi(s) \psi(\xi), \psi=\left(\mathcal{S}_{n}^{\perp}-\lambda_{n}\right)^{-1} F$, where by the smoothness improving theorems $\psi \in C^{\infty}(\bar{\omega})$. Therefore, $\widetilde{\psi}_{2}^{(n, m)} \in C^{\infty}(\bar{\Omega})$.

We substitute formulas (3.6), (3.7), (3.8), (3.11), (3.14), (3.15), (3.18), (3.21), (3.26) into problem (3.5) for $i=3$,

$$
\begin{gathered}
\left(-\Delta_{\xi}-\lambda_{n}\right) \psi_{3}^{(n, m)}=\lambda_{0}^{(n, m)} \psi_{1}^{(n, m)}+\lambda_{1}^{(n, m)} \psi_{0}^{(n, m)}+F_{3}^{(n, m)} \quad \text { in } \Omega, \quad \psi_{3}^{(n, m)}=0 \quad \text { on } \partial \Omega, \\
F_{3}^{(n, m)}=\widetilde{F}_{3}^{(n, m)}+\widetilde{\mathcal{F}} \Psi_{1}^{(n, m)} \phi_{n}-\Psi_{2}^{(n, m)}\left(\kappa_{1} \frac{\partial}{\partial \xi_{2}}-\kappa_{2} \frac{\partial}{\partial \xi_{3}}\right) \phi_{n}, \\
\widetilde{F}_{3}^{(n, m)}:=\left(\mathcal{F}_{1}-\lambda_{n} \mathrm{q}\right) \widetilde{\psi}_{2}^{(n, m)}+\frac{1}{2} \mathcal{F}_{2} \mathrm{q} \psi_{0}^{(n, m)}+\left(\mathcal{F}_{3}-\lambda_{0}^{(n, m)} \mathrm{q}\right) \psi_{0}^{(n, m)} .
\end{gathered}
$$

We again treat this problem as that for $\mathcal{S}$ depending on $s$, and the corresponding solvability condition is

$$
\lambda_{0}^{(n, m)}\left(\psi_{1}^{(n, m)}, \phi_{n}\right)_{L_{2}(\omega)}+\lambda_{1}^{(n, m)} \Psi_{0}^{(n, m)}+\left(F_{3}^{(n, m)}, \phi_{n}\right)_{L_{2}(\omega)}=0
$$

In the same way how equation (3.24) was derived we obtain

$$
\begin{aligned}
\left(\mathcal{L}_{n}-\lambda_{0}^{(n, m)}\right) \Psi_{1}^{(n, m)} & =\lambda_{1}^{(n, m)} \Psi_{0}^{(n, m)}+f_{3}^{(n, m)} \\
f_{3}^{(n, m)}(s): & =\left(\widetilde{F}_{3}^{(n, m)}(s, \cdot), \phi_{n}\right)_{L_{2}(\omega)}+\frac{1}{2} \lambda_{0}^{(n, m)} \Psi_{0}^{(n, m)}(s) \mathrm{q}_{n}(s) \\
\mathrm{q}_{n}(s): & =\left(\mathrm{q}(s, \cdot) \phi_{n}, \phi_{n}\right)_{L_{2}(\omega)}=\kappa_{1}(s)\left(\xi_{2} \phi_{n}, \phi_{n}\right)_{L_{2}(\omega)}-\kappa_{2}(s)\left(\xi_{3} \phi_{n}, \phi_{n}\right)_{L_{2}(\omega)} .
\end{aligned}
$$

Since $\lambda_{0}^{(n, m)}$ is an eigenvalue of $\mathcal{L}_{n}$, the solvability condition of the last equation is the orthogonality in $L_{2}\left(0, s_{0}\right)$ of its right hand side to the eigenfunctions associated with $\lambda_{0}^{(n, m)}$, i.e., it should be orthogonal to $\Psi_{0}^{(n, m)}$. It gives the formula for $\lambda_{1}^{(n, m)}$,

$$
\lambda_{1}^{(n, m)}=-\left(f_{3}^{(n, m)}, \Psi_{0}^{(n, m)}\right)_{L_{2}\left(0, s_{0}\right)}=-\left(\widetilde{F}_{3}^{(n, m)}, \psi_{0}^{(n, m)}\right)_{L_{2}(\Omega)}-\frac{1}{2} \lambda_{0}^{(n, m)}\left(\psi_{0}^{(n, m)}, \mathrm{q} \psi_{0}^{(n, m)}\right)_{L_{2}(\Omega)}
$$


Let us calculate the right hand side of this identity. Integrating by parts and employing (3.11), (3.13), (3.17), (3.20), we have

$$
\begin{aligned}
& \left(\left(\mathcal{F}_{1}-\mathrm{q} \lambda_{n}\right) \widetilde{\psi}_{2}^{(n, m)}, \psi_{0}^{(n, m)}\right)_{L_{2}(\Omega)}=\left(\widetilde{\psi}_{2}^{(n, m)},\left(\mathcal{F}_{1}-\mathrm{q} \lambda_{n}\right) \psi_{0}^{(n, m)}\right)_{L_{2}(\Omega)} \\
& \quad=-\frac{1}{2}\left(\widetilde{\psi}_{2}^{(n, m)},\left(\Delta_{\xi}+\lambda_{n}\right) \mathrm{q} \psi_{0}^{(n, m)}\right)_{L_{2}(\Omega)}=-\frac{1}{2}\left(\left(\Delta_{\xi}+\lambda_{n}\right) \widetilde{\psi}_{2}^{(n, m)}, \mathrm{q} \psi_{0}^{(n, m)}\right)_{L_{2}(\Omega)} \\
& \quad=\frac{1}{2}\left(\left(\widetilde{\mathcal{F}}+\lambda_{0}^{(n, m)}\right) \psi_{0}^{(n, m)}, \mathrm{q} \psi_{0}^{(n, m)}\right)_{L_{2}(\Omega)} \\
& \quad=\frac{1}{2}\left(\frac{1}{2}\left(\mathcal{F}_{1}-\mathrm{q} \lambda_{n}\right) \mathrm{q} \psi_{0}^{(n, m)}+\left(\mathcal{F}_{2}+\lambda_{0}^{(n, m)}\right) \psi_{0}^{(n, m)}, \mathrm{q} \psi_{0}^{(n, m)}\right)_{L_{2}(\Omega)} .
\end{aligned}
$$

Due to (3.8), (3.18)

$$
\left(\mathcal{F}_{1}-\mathrm{q} \lambda_{n}\right) \mathrm{q} \psi_{0}^{(n, m)}=-3 \mathrm{q}\left(\kappa_{1} \frac{\partial}{\partial \xi_{2}}-\kappa_{2} \frac{\partial}{\partial \xi_{3}}\right) \psi_{0}^{(n, m)}-\left(\kappa_{1}^{2}+\kappa_{2}^{2}\right) \psi_{0}^{(n, m)} .
$$

We multiply this equation by $\mathrm{q} \psi_{0}^{(n, m)}$ and integrate by parts,

$$
\begin{aligned}
\left(\left(\mathcal{F}_{1}-\mathrm{q} \lambda_{n}\right) \mathrm{q} \psi_{0}^{(n, m)}, \mathrm{q} \psi_{0}^{(n, m)}\right)_{L_{2}(\Omega)}= & -3\left(\mathrm{q}^{2}\left(\kappa_{1} \frac{\partial}{\partial \xi_{2}}-\kappa_{2} \frac{\partial}{\partial \xi_{3}}\right) \psi_{0}^{(n, m)}, \psi_{0}^{(n, m)}\right)_{L_{2}(\Omega)} \\
& -\left(\left(\kappa_{1}^{2}+\kappa_{2}^{2}\right) \psi_{0}^{(n, m)}, \mathrm{q} \psi_{0}^{(n, m)}\right)_{L_{2}(\Omega)} \\
= & -\frac{3}{2}\left(\mathrm{q}^{2},\left(\kappa_{1} \frac{\partial}{\partial \xi_{2}}-\kappa_{2} \frac{\partial}{\partial \xi_{3}}\right)\left(\psi_{0}^{(n, m)}\right)^{2}\right)_{L_{2}(\Omega)} \\
& -\left(\left(\kappa_{1}^{2}+\kappa_{2}^{2}\right) \psi_{0}^{(n, m)}, \mathrm{q} \psi_{0}^{(n, m)}\right)_{L_{2}(\Omega)} \\
= & 2\left(\left(\kappa_{1}^{2}+\kappa_{2}^{2}\right) \psi_{0}^{(n, m)}, \mathrm{q} \psi_{0}^{(n, m)}\right)_{L_{2}(\Omega)} .
\end{aligned}
$$

Substituting the identities obtained into (3.33), we arrive at

$$
\left(\left(\mathcal{F}_{1}-\mathrm{q} \lambda_{n}\right) \widetilde{\psi}_{2}^{(n, m)}, \psi_{0}^{(n, m)}\right)_{L_{2}(\Omega)}=\frac{1}{2}\left(\left(\mathcal{F}_{2}+\kappa_{1}^{2}+\kappa_{2}^{2}+\lambda_{0}^{(n, m)}\right) \psi_{0}^{(n, m)}, \mathrm{q} \psi_{0}^{(n, m)}\right)_{L_{2}(\Omega)} .
$$

It follows from (3.7) that

$$
\mathcal{F}_{3}=\mathrm{q} \mathcal{F}_{2}+\frac{\partial \mathrm{q}}{\partial s} \frac{\partial}{\partial s}+(\mathcal{R} \mathrm{q}) \kappa_{3} \frac{\partial}{\partial s}+\frac{\partial \mathrm{q}}{\partial s} \kappa_{3} \mathcal{R}+\kappa_{3}^{2}(\mathcal{R} \mathrm{q}) \mathcal{R} .
$$

We substitute this identity and (3.34) into (3.32) and integrate by parts,

$$
\begin{aligned}
\lambda_{1}^{(n, m)}= & -\frac{1}{2}\left(\left(\mathcal{F}_{2}+\kappa_{1}^{2}+\kappa_{2}^{2}\right) \psi_{0}^{(n, m)}, \mathrm{q} \psi_{0}^{(n, m)}\right)_{L_{2}(\Omega)}-\frac{1}{2}\left(\mathcal{F}_{2} \mathrm{q} \psi_{0}^{(n, m)}, \psi_{0}^{(n, m)}\right)_{L_{2}(\Omega)} \\
& -\left(\mathrm{q} \mathcal{F}_{2} \psi_{0}^{(n, m)}, \psi_{0}^{(n, m)}\right)_{L_{2}(\Omega)}-\left(\frac{\partial \mathrm{q}}{\partial s} \frac{\partial \psi_{0}^{(n, m)}}{\partial s}, \psi_{0}^{(n, m)}\right)_{L_{2}(\Omega)}-\left(\kappa_{3} \frac{\partial \psi_{0}^{(n, m)}}{\partial s} \mathcal{R} \mathrm{q}, \psi_{0}^{(n, m)}\right)_{L_{2}(\Omega)} \\
& -\left(\kappa_{3} \frac{\partial \mathrm{q}}{\partial s} \mathcal{R} \psi_{0}^{(n, m)}, \psi_{0}^{(n, m)}\right)_{L_{2}(\Omega)}-\left(\kappa_{3}^{2} \mathcal{R} \psi_{0}^{(n, m)}, \psi_{0}^{(n, m)} \mathcal{R} \mathrm{q}\right)_{L_{2}(\Omega)}
\end{aligned}
$$




$$
\begin{aligned}
= & -2\left(\mathcal{F}_{2} \psi_{0}^{(n, m)}, \mathrm{q} \psi_{0}^{(n, m)}\right)_{L_{2}(\Omega)}-\frac{1}{2}\left(\left(\kappa_{1}^{2}+\kappa_{2}^{2}\right) \psi_{0}^{(n, m)}, \mathrm{q} \psi_{0}^{(n, m)}\right)_{L_{2}(\Omega)} \\
& -\left(\frac{\partial \mathrm{q}}{\partial s} \frac{\partial \psi_{0}^{(n, m)}}{\partial s}, \psi_{0}^{(n, m)}\right)_{L_{2}(\Omega)}-\left(\kappa_{3} \frac{\partial \psi_{0}^{(n, m)}}{\partial s} \mathcal{R} \mathrm{q}, \psi_{0}^{(n, m)}\right)_{L_{2}(\Omega)} \\
& -\left(\kappa_{3} \frac{\partial \mathrm{q}}{\partial s} \mathcal{R} \psi_{0}^{(n, m)}, \psi_{0}^{(n, m)}\right)_{L_{2}(\Omega)}-\left(\kappa_{3}^{2} \mathcal{R} \psi_{0}^{(n, m)}, \psi_{0}^{(n, m)} \mathcal{R} \mathrm{q}\right)_{L_{2}(\Omega)}
\end{aligned}
$$

In view of (3.6), (3.24) we have

$$
\begin{aligned}
-\frac{1}{2}\left(\left(\kappa_{1}^{2}+\kappa_{2}^{2}\right) \psi_{0}^{(n, m)}, \mathrm{q} \psi_{0}^{(n, m)}\right)_{L_{2}(\Omega)} & -2\left(\mathcal{F}_{2} \psi_{0}^{(n, m)}, \mathrm{q} \psi_{0}^{(n, m)}\right)_{L_{2}(\Omega)} \\
=2\left(\left(\lambda_{0}^{(n, m)}-C_{n}(\omega) \kappa_{3}^{2}\right) \psi_{0}^{(n, m)}, \mathrm{q} \psi_{0}^{(n, m)}\right)_{L_{2}(\Omega)}-2\left(\mathcal{R} \kappa_{3} \frac{\partial \psi_{0}^{(n, m)}}{\partial s}, \mathrm{q} \psi_{0}^{(n, m)}\right)_{L_{2}(\Omega)} & \\
& -2\left(\frac{\partial}{\partial s} \kappa_{3} \mathcal{R} \psi_{0}^{(n, m)}, \mathrm{q} \psi_{0}^{(n, m)}\right)_{L_{2}(\Omega)}-2\left(\kappa_{3}^{2} \mathcal{R}^{2} \psi_{0}^{(n, m)}, \mathrm{q} \psi_{0}^{(n, m)}\right)_{L_{2}(\Omega)} .
\end{aligned}
$$

We integrate by parts employing (3.8),

$$
\begin{aligned}
- & 2\left(\mathcal{R}_{3} \frac{\partial \psi_{0}^{(n, m)}}{\partial s}, \mathrm{q} \psi_{0}^{(n, m)}\right)_{L_{2}(\Omega)}-2\left(\frac{\partial}{\partial s} \kappa_{3} \mathcal{R} \psi_{0}^{(n, m)}, \mathrm{q} \psi_{0}^{(n, m)}\right)_{L_{2}(\Omega)} \\
& -\left(\kappa_{3} \frac{\partial \psi_{0}^{(n, m)}}{\partial s} \mathcal{R} \mathrm{q}, \psi_{0}^{(n, m)}\right)_{L_{2}(\Omega)}-\left(\kappa_{3} \frac{\partial \mathrm{q}}{\partial s} \mathcal{R} \psi_{0}^{(n, m)}, \psi_{0}^{(n, m)}\right)_{L_{2}(\Omega)} \\
& =2\left(\kappa_{3} \frac{\partial \psi_{0}^{(n, m)}}{\partial s}, \mathcal{R} \mathrm{q} \psi_{0}^{(n, m)}\right)_{L_{2}(\Omega)}+2\left(\kappa_{3} \mathcal{R} \psi_{0}^{(n, m)}, \frac{\partial}{\partial s} \mathrm{q} \psi_{0}^{(n, m)}\right)_{L_{2}(\Omega)} \\
& -\left(\kappa_{3} \frac{\partial \psi_{0}^{(n, m)}}{\partial s} \mathcal{R} \mathrm{q}, \psi_{0}^{(n, m)}\right)_{L_{2}(\Omega)}-\left(\kappa_{3} \frac{\partial \mathrm{q}}{\partial s} \mathcal{R} \psi_{0}^{(n, m)}, \psi_{0}^{(n, m)}\right)_{L_{2}(\Omega)} \\
= & \left(\kappa_{3} \frac{\partial \psi_{0}^{(n, m)}}{\partial s}, \psi_{0}^{(n, m)} \mathcal{R} \mathrm{q}\right)_{L_{2}(\Omega)}+\left(\kappa_{3} \frac{\partial \mathrm{q}}{\partial s} \mathcal{R} \psi_{0}^{(n, m)}, \psi_{0}^{(n, m)}\right)_{L_{2}(\Omega)}+4\left(\kappa_{3} \mathrm{q} \mathcal{R} \psi_{0}^{(n, m)}, \frac{\partial \psi_{0}^{(n, m)}}{\partial s}\right)_{L_{2}(\Omega)} \\
= & \frac{1}{2}\left(\kappa_{3} \mathcal{R} \mathrm{q}, \frac{\partial}{\partial s}\left(\psi_{0}^{(n, m)}\right)^{2}\right)_{L_{2}(\Omega)}+\frac{1}{2}\left(\kappa_{3} \frac{\partial \mathrm{q}}{\partial s}, \mathcal{R}\left(\psi_{0}^{(n, m)}\right)^{2}\right)_{L_{2}(\Omega)}+\left(\kappa_{3} \mathrm{q} \mathcal{R} \phi_{n}^{2}, \frac{\partial}{\partial s}\left(\Psi_{0}^{(n, m)}\right)^{2}\right)_{L_{2}(\Omega)} \\
= & -\frac{1}{2}\left(\frac{\partial}{\partial s} \kappa_{3} \mathcal{R} \mathrm{q},\left(\psi_{0}^{(n, m)}\right)^{2}\right)_{L_{2}(\Omega)}-\frac{1}{2}\left(\mathcal{R} \kappa_{3} \frac{\partial \mathrm{q}}{\partial s},\left(\psi_{0}^{(n, m)}\right)^{2}\right)_{L_{2}(\Omega)}+\left(\phi_{n}^{2}\left(\Psi_{0}^{(n, m)}\right)^{2}, \frac{\partial}{\partial s} \kappa_{3} \mathcal{R} \mathrm{q}\right)_{L_{2}(\Omega)} \\
= & \frac{1}{2}\left(\psi_{0}^{(n, m)}, \psi_{0}^{(n, m)} \frac{\partial}{\partial s} \kappa_{3} \mathcal{R} \mathrm{q}\right)_{L_{2}(\Omega)}-\frac{1}{2}\left(\psi_{0}^{(n, m)}, \psi_{0}^{(n, m)} \kappa_{3} \mathcal{R} \frac{\partial \mathrm{q}}{\partial s}\right)_{L_{2}(\Omega)} \\
= & \frac{1}{2}\left(\psi_{0}^{(n, m)}, \psi_{0}^{(n, m)} \kappa_{3}^{\prime} \mathcal{R} \mathrm{q}\right)_{L_{2}(\Omega)}^{.}
\end{aligned}
$$

In the same fashion we obtain

$$
-\left(\frac{\partial \mathrm{q}}{\partial s} \frac{\partial \psi_{0}^{(n, m)}}{\partial s}, \psi_{0}^{(n, m)}\right)_{L_{2}(\Omega)}=\frac{1}{2}\left(\psi_{0}^{(n, m)}, \frac{\partial^{2} \mathrm{q}}{\partial s^{2}} \psi_{0}^{(n, m)}\right)_{L_{2}(\Omega)} .
$$


Employing the identity $\mathcal{R}^{2} \mathrm{q}=-\mathrm{q}$, we get

$$
\begin{aligned}
& -\left(\kappa_{3}^{2} \mathcal{R} \psi_{0}^{(n, m)}, \psi_{0}^{(n, m)} \mathcal{R} \mathrm{q}\right)_{L_{2}(\Omega)}-2\left(\kappa_{3}^{2} \mathcal{R}^{2} \psi_{0}^{(n, m)}, \mathrm{q} \psi_{0}^{(n, m)}\right)_{L_{2}(\Omega)} \\
& =-\left(\kappa_{3}^{2} \mathcal{R} \psi_{0}^{(n, m)}, \psi_{0}^{(n, m)} \mathcal{R} \mathrm{q}\right)_{L_{2}(\Omega)}+2\left(\kappa_{3}^{2} \mathcal{R} \psi_{0}^{(n, m)}, \mathcal{R} \mathrm{q} \psi_{0}^{(n, m)}\right)_{L_{2}(\Omega)} \\
& =2\left(\kappa_{3}^{2} \mathcal{R} \psi_{0}^{(n, m)}, \mathrm{q} \mathcal{R} \psi_{0}^{(n, m)}\right)_{L_{2}(\Omega)}+\left(\kappa_{3}^{2} \mathcal{R} \psi_{0}^{(n, m)}, \psi_{0}^{(n, m)} \mathcal{R} \mathrm{q}\right)_{L_{2}(\Omega)} \\
& =2\left(\kappa_{3}^{2} \mathcal{R} \psi_{0}^{(n, m)}, \mathrm{q} \mathcal{R} \psi_{0}^{(n, m)}\right)_{L_{2}(\Omega)}+\frac{1}{2}\left(\kappa_{3}^{2} \mathcal{R}\left(\psi_{0}^{(n, m)}\right)^{2}, \mathcal{R} \mathrm{q}\right)_{L_{2}(\Omega)} \\
& =2\left(\kappa_{3}^{2} \mathcal{R} \psi_{0}^{(n, m)}, \mathrm{q} \mathcal{R} \psi_{0}^{(n, m)}\right)_{L_{2}(\Omega)}-\frac{1}{2}\left(\kappa_{3}^{2} \psi_{0}^{(n, m)}, \psi_{0}^{(n, m)} \mathcal{R}^{2} \mathrm{q}\right)_{L_{2}(\Omega)} \\
& =2\left(\kappa_{3}^{2} \mathcal{R} \psi_{0}^{(n, m)}, \mathrm{q} \mathcal{R} \psi_{0}^{(n, m)}\right)_{L_{2}(\Omega)}+\frac{1}{2}\left(\kappa_{3}^{2} \psi_{0}^{(n, m)}, \mathrm{q} \psi_{0}^{(n, m)}\right)_{L_{2}(\Omega)} .
\end{aligned}
$$

We substitute the identities obtained and (3.37), (3.38), (3.39) into (3.35) that leads us to formula (1.6) for $\lambda_{1}^{(n, m)}$.

Let $V_{n, m}$ be the orthogonal complement to $\left\{\Psi_{0}^{(n, m)}\right\}$ in $L_{2}\left(0, s_{0}\right)$, and $\mathcal{L}_{n, m}^{\perp}$ be the restriction of $\mathcal{L}_{n}$ to $V_{n, m} \cap W_{2,0}^{2}\left(0, s_{0}\right)$. The operator $\left(\mathcal{L}_{n, m}^{\perp}-\lambda_{0}^{(n, m)}\right)^{-1}$ is well-defined and bounded as that from $V_{n, m}$ into $V_{n, m} \cap$ $W_{2,0}^{2}\left(0, s_{0}\right)$.

The orthogonality condition (3.32) means that the right hand side of (3.30) is orthogonal to $\Psi_{0}^{(n, m)}$. Thus, $\lambda_{1}^{(n, m)} \Psi_{0}^{(n, m)}+f_{3}^{(n, m)} \in V_{n, m}$. In view of this fact we can choose a solution to (3.30) as

$$
\Psi_{1}^{(n, m)}=\left(\mathcal{L}_{n, m}^{\perp}-\lambda_{0}^{(n, m)}\right)^{-1}\left(\lambda_{1}^{(n, m)} \Psi_{0}^{(n, m)}+f_{3}^{(n, m)}\right)+C \Psi_{0}^{(n, m)},
$$

where $C$ is an arbitrary constant. We could choose it zero, but as we will see in what follows the most convenient way is to choose $C$ by the orthogonality condition

$$
\left(\psi_{1}^{(n, m)}, \psi_{0}^{(n, m)}\right)_{L_{2}(\Omega)}=0
$$

By (3.15), (3.31) and by the fact that the range of $\left(\mathcal{L}_{n, m}^{\perp}-\lambda_{0}^{(n, m)}\right)^{-1}$ is orthogonal to $\left\{\Psi_{0}^{(n, m)}\right\}$ it yields

$$
C=-\frac{1}{2}\left(\psi_{0}^{(n, m)}, \mathrm{q} \psi_{0}^{(n, m)}\right)_{L_{2}(\Omega)}=-\frac{1}{2}\left(\Psi_{0}^{(n, m)}, \mathrm{q}_{n} \Psi_{0}^{(n, m)}\right)_{L_{2}\left(0, s_{0}\right)}
$$

and

$$
\Psi_{1}^{(n, m)}=\left(\mathcal{L}_{n, m}^{\perp}-\lambda_{0}^{(n, m)}\right)^{-1}\left(\lambda_{1}^{(n, m)} \Psi_{0}^{(n, m)}+f_{3}^{(n, m)}\right)-\frac{1}{2}\left(\Psi_{0}^{(n, m)}, \mathrm{q}_{n} \Psi_{0}^{(n, m)}\right)_{L_{2}\left(0, s_{0}\right)} \Psi_{0}^{(n, m)}
$$

By the smoothness improving theorems $\Psi_{1}^{(n, m)} \in C^{\infty}\left[0, s_{0}\right]$.

Equations (3.29), (3.30) being satisfied, the right hand side of the equation in (3.27) is orthogonal to $\phi_{n}$, i.e.,

$$
\lambda_{0}^{(n, m)} \psi_{1}^{(n, m)}+\lambda_{1}^{(n, m)} \psi_{0}^{(n, m)}+F_{3}^{(n, m)} \in V_{n}
$$

By (3.25), (3.28) it yields

$$
\lambda_{0}^{(n, m)} \psi_{1}^{(n, m)}+\lambda_{1}^{(n, m)} \psi_{0}^{(n, m)}+\widetilde{F}_{3}^{(n, m)}+\widetilde{\mathcal{F}} \Psi_{1}^{(n, m)} \phi_{n} \in V_{n} .
$$


In view of this fact we can choose a solution to (3.27) as

$$
\begin{aligned}
\psi_{3}^{(n, m)}(s, \xi) & =\widetilde{\psi}_{3}^{(n, m)}(s, \xi)+\frac{1}{2} \Psi_{2}^{(n, m)}(s) \phi_{n}(\xi) \mathrm{q}(s, \xi)+\Psi_{3}^{(n, m)}(s) \phi_{n}(\xi), \\
\widetilde{\psi}_{3}^{(n, m)} & :=\left(\mathcal{S}_{n}^{\perp}-\lambda_{n}\right)^{-1}\left(\lambda_{0}^{(n, m)} \psi_{1}^{(n, m)}+\lambda_{1}^{(n, m)} \psi_{0}^{(n, m)}+\widetilde{F}_{3}^{(n, m)}+\widetilde{\mathcal{F}} \Psi_{1}^{(n, m)} \phi_{n}\right),
\end{aligned}
$$

where the function $\Psi_{3}^{(n, m)}$ is unknown and should satisfy the boundary conditions

$$
\Psi_{3}^{(n, m)}(0)=\Psi_{3}^{(n, m)}\left(s_{0}\right)=0,
$$

and $\widetilde{\psi}_{3}^{(n, m)} \in C^{\infty}(\bar{\Omega})$. This smoothness is proved in the same way as for $\widetilde{\psi}_{2}^{(n, m)}$.

The remaining problems for $\psi_{i}, i \geqslant 4$, are solved in the same way as for $i=1,2,3$. Namely, the solvability condition of these problems is the orthogonality of the right hand side to $\phi_{n}$ in $L_{2}(\omega)$ for each $s \in\left(0, s_{0}\right)$. In its turn, these condition imply the equations for $\Psi_{i}$. The solvability conditions of these equations imply the formulas for $\lambda_{i}$. The result of this recurrent procedure is formulated in:

Lemma 3.1. There exist solutions to problems (3.5) given by the identities

$$
\psi_{i}^{(n, m)}(s, \xi)=\widetilde{\psi}_{i}^{(n, m)}(s, \xi)+\frac{1}{2} \Psi_{i-1}^{(n, m)}(s) \mathrm{q}(s, \xi) \phi_{n}(\xi)+\Psi_{i}^{(n, m)}(s) \phi_{n}(\xi), \quad i \geqslant 0 .
$$

The functions $\widetilde{\psi}_{i}^{(n, m)} \in C^{\infty}(\bar{\Omega}), \Psi_{i}^{(n, m)} \in C^{\infty}\left[0, s_{0}\right]$ read as follows,

$$
\begin{aligned}
& \widetilde{\psi}_{i}^{(n, m)}(s, \xi)=0, \quad i \leqslant 1, \\
& \widetilde{\psi}_{i}^{(n, m)}(s, \xi)=\left(\mathcal{S}_{n}^{\perp}-\lambda_{n}\right)^{-1}\left(\widetilde{F}_{i}^{(n, m)}+\widetilde{\mathcal{F}} \Psi_{i-2}^{(n, m)} \phi_{n}+\sum_{j=2}^{i} \lambda_{j-2}^{(n, m)} \psi_{i-j}^{(n, m)}\right), \quad i \geqslant 2, \\
& \widetilde{F}_{i}^{(n, m)}=0, \quad i \leqslant 2, \\
& \widetilde{F}_{i}^{(n, m)}=\left(\mathcal{F}_{1}-\lambda_{n} \mathrm{q}\right) \widetilde{\psi}_{i-1}^{(n, m)}+\mathcal{F}_{2}\left(\widetilde{\psi}_{i-2}^{(n, m)}+\frac{1}{2} \Psi_{i-3}^{(n, m)} \mathrm{q} \phi_{n}\right)+\sum_{j=3}^{i}\left(\mathcal{F}_{j}-\lambda_{j-3}^{(n, m)} \mathrm{q}\right) \psi_{i-j}^{(n, m)}, \quad i \geqslant 3, \\
& \Psi_{i}^{(n, m)}=\left(\mathcal{L}_{n, m}^{\perp}-\lambda_{0}^{(n, m)}\right)^{-1}\left(f_{i+2}^{(n, m)}+\sum_{j=1}^{i} \lambda_{j}^{(n, m)} \Psi_{i-j}^{(n, m)}\right) \\
& \quad-\frac{1}{2}\left(\Psi_{i-1}^{(n, m)}, \mathrm{q}_{n} \Psi_{0}^{(n, m)}\right)_{L_{2}\left(0, s_{0}\right)} \Psi_{0}^{(n, m)}, \quad i \geqslant 1, \\
& f_{i}^{(n, m)}=\left(\widetilde{F}_{i}, \psi_{0}^{(n, m)}\right)_{L_{2}(\Omega)}+\frac{1}{2} \sum_{j=0}^{i-3} \lambda_{j}^{(n, m)} \Psi_{i-j-3}^{(n, m)} \mathrm{q}_{n}, \quad i \geqslant 3,
\end{aligned}
$$

where $\lambda_{-2}^{(n, m)}$ and $\lambda_{-1}^{(n, m)}$ are determined by (3.8), (3.14), $\lambda_{0}^{(n, m)}$ is an eigenvalue of $\mathcal{L}_{n}$ with the associated eigenfunction $\Psi_{0}^{(n, m)}$, and

$$
\lambda_{i}^{(n, m)}=-\left(\widetilde{F}_{i+2}^{(n, m)}, \psi_{0}^{(n, m)}\right)_{L_{2}(\Omega)}-\frac{1}{2} \lambda_{0}^{(n, m)}\left(\Psi_{i-1}^{(n, m)} \phi_{n}, \mathrm{q} \psi_{0}^{(n, m)}\right)_{L_{2}(\Omega)}, \quad i \geqslant 1 .
$$

The identities

$$
\left(\psi_{i}^{(n, m)}, \psi_{0}^{(n, m)}\right)_{L_{2}(\Omega)}=0, \quad i \geqslant 1
$$

hold true. 
Remark 3.2. Formula (3.42) involves the term $\mathcal{F}_{2} \widetilde{\psi}_{i-2}^{(n, m)}$. It is zero for $i \geqslant 3$, and this is why it is absent in (3.19), (3.28). At the same time, it is easy to see that it comes from the term $\left(\mathcal{F}_{2}-\lambda_{j-3}^{(n, m)}\right) \psi_{i-2}^{(n, m)}$ in $(3.6)$, when $\psi_{i-2}^{(n, m)}$ is taken in accordance with (3.41).

Remark 3.3. The identities (3.45) are analogues of condition (3.40) and they imply the second term in formulas (3.43) for $\Psi_{i}^{(n, m)}$, where we used that

$$
\left(\widetilde{\psi}_{i}^{(n, m)}, \psi_{0}^{(n, m)}\right)_{L_{2}(\Omega)}=0 .
$$

This identity is valid since the range of $\left(\mathcal{S}_{n}-\lambda_{n}\right)^{-1}$ is orthogonal to $\phi_{n}$ in $L_{2}(\omega)$.

Given any $N \geqslant 1$, we denote

$$
\lambda_{\varepsilon, N}^{(n, m)}:=\varepsilon^{-2} \lambda_{n}+\sum_{i=0}^{N-2} \varepsilon^{i} \lambda_{i}^{(n, m)}, \quad \psi_{\varepsilon, N}^{(n, m)}(s, \xi):=\sum_{i=0}^{N} \varepsilon^{i} \psi_{i}^{(n, m)}(s, \xi) .
$$

The next lemma follows directly from Lemma 3.1.

Lemma 3.4. The function $\psi_{\varepsilon, N}^{(n, m)} \in C^{\infty}(\bar{\Omega})$ and the number $\lambda_{\varepsilon, N}^{(n, m)}$ satisfy the equation

$$
\left(\widehat{\mathcal{H}}_{\varepsilon}-\lambda_{\varepsilon, N}^{(n, m)} \mathrm{p}_{\varepsilon}\right) \psi_{\varepsilon, N}^{(n, m)}=h_{\varepsilon, N}^{(n, m)},
$$

where the right-hand side obeys the inequality

$$
\left\|h_{\varepsilon, N}^{(n, m)}\right\|_{C^{k}(\bar{\Omega})} \leqslant C_{N, k}^{(n, m)} \varepsilon^{N-1},
$$

with constants $C_{N, k}^{(n, m)}>0$ independent of $\varepsilon$.

We proceed to the justification of formal asymptotics (3.1), (3.2). We use the standard approach based on Lemmas 12 and 13 from [15]. More precisely, we use these lemmas in the formulation presented in Lemma 1.1 in [13], Chapter III, Section 1.1. For the convenience of the reader below we give the mentioned lemma.

Lemma 3.5. Let $\mathcal{A}: H \rightarrow H$ be a continuous linear compact self-adjoint operator in a Hilbert space $H$. Suppose that there exist a real $\mu>0$ and a vector $u \in H$, such that $\|u\|_{H}=1$ and

$$
\|\mathcal{A} u-\mu u\|_{H} \leqslant \alpha, \quad \alpha=\text { const. }>0 .
$$

Then there exists an eigenvalue $\mu_{i}$ of operator $\mathcal{A}$ such that

$$
\left|\mu_{i}-\mu\right| \leqslant \alpha .
$$

Moreover, for any $d>\alpha$ there exists a vector $\bar{u}$ such that

$$
\|u-\bar{u}\|_{H} \leqslant 2 \alpha d^{-1}, \quad\|\bar{u}\|_{H}=1,
$$

and $\bar{u}$ is a linear combination of the eigenvectors of the operator $\mathcal{A}$ corresponding to the eigenvalues of $\mathcal{A}$ from the segment $[\mu-d, \mu+d]$.

Let $\varepsilon$ be small enough. Denote $\widehat{\psi}_{\varepsilon, N}^{(n, m)}:=\mathrm{p}_{\varepsilon}^{1 / 2} \psi_{\varepsilon, N}^{(n, m)}$. We remind that the function $\mathrm{p}_{\varepsilon}$ was introduced in (2.6), while the function q was defined in Theorem 1.1.

We rewrite (3.47) as

$$
\mathrm{p}_{\varepsilon}^{-1 / 2} \widehat{\mathcal{H}}_{\varepsilon} \mathrm{p}_{\varepsilon}^{-1 / 2} \widehat{\psi}_{\varepsilon, N}^{(n, m)}=\lambda_{\varepsilon, N}^{(n, m)} \widehat{\psi}_{\varepsilon, N}^{(n, m)}+\mathrm{p}_{\varepsilon}^{-1 / 2} h_{\varepsilon, N}^{(n, m)}
$$


Identities (2.3), (2.4), (2.5) imply that the operator $\widehat{\mathcal{H}}_{\varepsilon}$ is self-adjoint and non-negative. The same is obviously true for $\mathrm{p}_{\varepsilon}^{-1 / 2} \widehat{\mathcal{H}}_{\varepsilon} \mathrm{p}_{\varepsilon}^{-1 / 2}$. Let $\delta>0$ be a positive number. Then the operator $\mathcal{A}_{\varepsilon}:=\left(1+\delta \mathrm{p}_{\varepsilon}^{-1 / 2} \widehat{\mathcal{H}}_{\varepsilon} \mathrm{p}_{\varepsilon}^{-1 / 2}\right)^{-1}$ is well-defined as an operator in $L_{2}(\Omega)$, is bounded and self-adjoint, and satisfies the estimate

$$
\left\|\mathcal{A}_{\varepsilon}\right\| \leqslant 1 .
$$

As it follows from (2.4), for any $f \in L_{2}(\Omega)$ the function $v=\mathcal{A}_{\varepsilon} f$ is a generalized solution to the boundary value problem

$$
\begin{aligned}
-\delta \mathrm{p}_{\varepsilon}^{-1 / 2} \sum_{i, j=1}^{3} \frac{\partial}{\partial \xi_{i}} \widetilde{A}_{i j}^{(\varepsilon)} \frac{\partial}{\partial \xi_{j}} \mathrm{p}_{\varepsilon}^{-1 / 2} v+v=f \quad \text { in } \Omega, \quad v=0 \quad \text { on } \partial \Omega . \\
\xi_{1}:=s, \quad \widetilde{A}_{11}^{(\varepsilon)}:=A_{11}^{(\varepsilon)}, \quad \widetilde{A}_{i 1}^{(\varepsilon)}:=\widetilde{A}_{1 i}^{(\varepsilon)}:=\varepsilon^{-1} A_{i 1}^{(\varepsilon)}, \quad \widetilde{A}_{i j}^{(\varepsilon)}:=\varepsilon^{-2} A_{i j}^{(\varepsilon)}, \quad i, j=2,3 .
\end{aligned}
$$

Hence, operator $\mathcal{A}_{\varepsilon}$ is also bounded as that from $L_{2}(\Omega)$ into $W_{2}^{1}(\Omega)$. In view of the compact embedding of $W_{2}^{1}(\Omega)$ in $L_{2}(\Omega)$ operator $\mathcal{A}_{\varepsilon}$ is compact as that in $L_{2}(\Omega)$.

We rewrite (3.49) as

$$
\left(1+\delta \lambda_{\varepsilon, N}^{(n, m)}\right)^{-1} \widehat{\psi}_{\varepsilon, N}^{(n, m)}=\mathcal{A}_{\varepsilon} \widehat{\psi}_{\varepsilon, N}^{(n, m)}+\delta \widehat{h}_{\varepsilon, N}^{(n, m)}, \quad \widehat{h}_{\varepsilon, N}^{(n, m)}:=\left(1+\delta \lambda_{\varepsilon, N}^{(n, m)}\right)^{-1} \mathcal{A}_{\varepsilon} \mathrm{p}_{\varepsilon}^{-1 / 2} h_{\varepsilon, N}^{(n, m)} .
$$

In view of definition (3.46) of $\lambda_{\varepsilon, N}^{(n, m)}$ we can choose small $\delta=\delta(\varepsilon, N, m, n)$ such that

$$
\delta\left|\lambda_{\varepsilon, N}^{(n, m)}\right| \leqslant \frac{1}{2}, \quad \frac{1}{2} \leqslant 1+\delta \lambda_{\varepsilon, N}^{(n, m)} \leqslant \frac{3}{2}
$$

By definition (2.6) of $\mathrm{p}_{\varepsilon}$ and smoothness $\mathrm{q} \in C^{\infty}(\bar{\Omega})$, for sufficiently small $\varepsilon$ we can always assume the estimate

$$
\frac{1}{2} \leqslant\left|\mathrm{p}_{\varepsilon}\right| \leqslant \frac{3}{2}
$$

Hence, by the definition of $\widehat{\psi}_{\varepsilon, N}^{(n, m)}$

$$
\left\|\widehat{\psi}_{\varepsilon, N}^{(n, m)}\right\|_{L_{2}(\Omega)} \geqslant \frac{1}{2}\left\|\psi_{\varepsilon, N}^{(n, m)}\right\|_{L_{2}(\Omega)} .
$$

Employing (3.45) and the normalization of $\phi_{n}$ and $\Psi_{0}^{(n, m)}$, we obtain

$$
\begin{aligned}
\left\|\psi_{\varepsilon, N}^{(n, m)}\right\|_{L_{2}(\Omega)}^{2} & =\left\|\psi_{0}^{(n, m)}\right\|_{L_{2}(\Omega)}^{2}+2 \sum_{i=1}^{N} \varepsilon^{i}\left(\psi_{i}^{(n, m)}, \psi_{0}^{(n, m)}\right)_{L_{2}(\Omega)}+\left\|\sum_{i=1}^{N} \varepsilon^{i} \psi_{i}^{(n, m)}\right\|_{L_{2}(\Omega)}^{2} \\
& =\left\|\psi_{0}^{(n, m)}\right\|_{L_{2}(\Omega)}^{2}+\left\|\sum_{i=1}^{N} \varepsilon^{i} \psi_{i}^{(n, m)}\right\|_{L_{2}(\Omega)}^{2} \geqslant 1 .
\end{aligned}
$$

Remark 3.6. We note that we introduced the orthogonality conditions (3.45) to guarantee the last estimate for $\left\|\psi_{\varepsilon, N}^{(n, m)}\right\|_{L_{2}(\Omega)}^{2}$ uniformly in $\varepsilon, N, n$, and $m$.

The estimates (3.53) and (3.54) yield

$$
\left\|\widehat{\psi}_{\varepsilon}^{(n, m)}\right\|_{L_{2}(\Omega)} \geqslant \frac{1}{2} .
$$

Bearing in mind this estimate and choosing if needed $\delta$ small enough, by (3.48) with $k=0,(3.50),(3.54)$ we get

$$
\frac{\delta\left\|\widehat{h}_{\varepsilon, N}^{(n, m)}\right\|_{L_{2}(\Omega)}}{\left\|\widehat{\psi}_{\varepsilon, N}^{(n, m)}\right\|_{L_{2}(\Omega)}} \leqslant \widehat{C}_{N}^{(n, m)} \delta \varepsilon^{N-1} \leqslant \frac{1}{6},
$$

where $\widehat{C}_{N}^{(n, m)}$ are some constants independent of $\varepsilon$. 
We apply Lemma 3.5 with

$$
H=L_{2}(\Omega), \quad \mathcal{A}=\mathcal{A}_{\varepsilon}, \quad \mu=\left(1+\delta \lambda_{\varepsilon, N}^{(n, m)}\right)^{-1}, \quad u=\frac{\widehat{\psi}_{\varepsilon, N}^{(n, m)}}{\left\|\widehat{\psi}_{\varepsilon, N}^{(n, m)}\right\|_{L_{2}(\Omega)}}, \quad \alpha=\widehat{C}_{N}^{(n, m)} \delta \varepsilon^{N-1}
$$

and conclude that there exists an eigenvalue $\mu_{\varepsilon, N}^{(n, m)}$ of $\mathcal{A}_{\varepsilon}$ such that

$$
\left|\mu_{\varepsilon, N}^{(n, m)}-\left(1+\delta \lambda_{\varepsilon, N}^{(n, m)}\right)^{-1}\right| \leqslant \widehat{C}_{N}^{(n, m)} \delta \varepsilon^{N-1}
$$

It is clear that $\widetilde{\lambda}_{\varepsilon, N}^{(n, m)}:=\left(\left(\mu_{\varepsilon, N}^{(n, m)}\right)^{-1}-1\right) \delta^{-1}$ is an eigenvalue of $\mathrm{p}_{\varepsilon}^{-1 / 2} \widehat{\mathcal{H}}_{\varepsilon} \mathrm{p}_{\varepsilon}^{-1 / 2}$. It follows from (3.57), (3.51), (3.55) that

$$
\frac{1}{1+\delta \widetilde{\lambda}_{\varepsilon, N}^{(n, m)}} \geqslant \frac{1}{1+\delta \lambda_{\varepsilon, N}^{(n, m)}}-\widehat{C}_{N}^{(n, m)} \varepsilon^{N-1} \delta \geqslant \frac{1}{2}, \quad 1+\delta \widetilde{\lambda}_{\varepsilon, N}^{(n, m)} \leqslant 2 .
$$

The last inequality and (3.51), (3.57) imply

$$
\begin{gathered}
\left|\left(1+\delta \widetilde{\lambda}_{\varepsilon, N}^{(n, m)}\right)-\left(1+\delta \lambda_{\varepsilon, N}^{(n, m)}\right)\right| \leqslant \widehat{C}_{N}^{(n, m)} \delta \varepsilon^{N-1}\left|1+\delta \widetilde{\lambda}_{\varepsilon, N}^{(n, m)}\right|\left|1+\delta \lambda_{\varepsilon, N}^{(n, m)}\right|, \\
\left|\widetilde{\lambda}_{\varepsilon, N}^{(n, m)}-\lambda_{\varepsilon, N}^{(n, m)}\right| \leqslant 3 \widehat{C}_{N}^{(n, m)} \varepsilon^{N-1} .
\end{gathered}
$$

By $\varepsilon_{N}^{(n, m)}$ we denote a monotonically decreasing (in $N$ ) sequence such that

$$
\widehat{C}_{N}^{(n, m)} \varepsilon \leqslant \widehat{C}_{N-1}^{(n, m)} \text { as } \varepsilon \leqslant \varepsilon_{N}^{(n, m)}
$$

Letting

$$
\lambda^{(n, m)}(\varepsilon):=\widetilde{\lambda}_{\varepsilon, N}^{(n, m)} \text { for } \quad \varepsilon \in\left[\varepsilon_{N}^{(n, m)}, \varepsilon_{N+1}^{(n, m)}\right),
$$

employing (3.58), and taking into account the fact that $N$ is arbitrary, we see that the eigenvalue $\lambda^{(n, m)}(\varepsilon)$ of $\mathrm{p}_{\varepsilon}^{-1 / 2} \widehat{\mathcal{H}}_{\varepsilon} \mathrm{p}_{\varepsilon}^{-1 / 2}$ has the asymptotic expansion (1.5). To complete the proof it remains to note that the eigenvalues of $\mathrm{p}_{\varepsilon}^{-1 / 2} \widehat{\mathcal{H}}_{\varepsilon} \mathrm{p}_{\varepsilon}^{-1 / 2}$ coincide with those of $\mathcal{H}_{\varepsilon}$.

\section{Proof of Theorem 1.2}

We begin the proof with the result of Theorem 4.4 in [3]. Namely, item (ii) of this theorem says that given any $M>0$, there exists $\varepsilon_{0}(M)>0$ so that for all $\varepsilon<\varepsilon_{0}$ the first $M$ eigenvalues $\lambda_{m}(\varepsilon), m=1, \ldots, M$, of $\mathcal{H}_{\varepsilon}$ taken counting multiplicities satisfy the asymptotics

$$
\lambda_{m}(\varepsilon)=\varepsilon^{-2} \lambda_{1}+\lambda_{0}^{(1, m)}+o(1), \quad m=1, \ldots, M .
$$

Since the eigenvalues $\lambda_{0}^{(1, m)}$ of $\mathcal{L}_{1}$ are simple, the same is true for the eigenvalues $\lambda_{m}(\varepsilon)$.

It follows from (4.1) that there exists a fixed number $\theta>0$ so that for each $m=1, \ldots, M$, and all $\varepsilon<\varepsilon_{0}(M)$ the interval

$$
\left(\varepsilon^{-2} \lambda_{1}+\lambda_{0}^{(1, m)}-\theta, \varepsilon^{-2} \lambda_{1}+\lambda_{0}^{(1, m)}+\theta\right)
$$

contains exactly one eigenvalue of $\mathcal{H}_{\varepsilon}$ which is $\lambda_{m}(\varepsilon)$. In accordance with Theorem 1.1 the eigenvalues $\lambda^{(1, m)}(\varepsilon)$ of $\mathcal{H}_{\varepsilon}$ satisfy the same asymptotics as $\lambda_{m}(\varepsilon)$. Hence, for sufficiently small $\varepsilon_{0}(M)$ and all $m=1, \ldots, M$ each of the intervals (4.2) contains the eigenvalue $\lambda^{(1, m)}(\varepsilon)$. Therefore, $\lambda^{(1, m)}(\varepsilon)=\lambda_{m}(\varepsilon)$, and it proves the statement of the theorem on the eigenvalues. 
To prove the statement on the eigenfunctions, we adopt the same notations as in the proof of Theorem 1.1. We again apply Lemma 3.5 with $(3.56)$ and we take $d=\varepsilon^{N / 2}$. Then there exists a linear combination $\widetilde{\psi}_{\varepsilon, N}^{(1, m)}$ of the eigenfunctions associated with the eigenvalues of $\mathcal{A}_{\varepsilon}$ lying in the segment

$$
\left[\left(1+\delta \lambda_{\varepsilon, N}^{(1, m)}\right)^{-1}-\varepsilon^{N / 2},\left(1+\delta \lambda_{\varepsilon, N}^{(1, m)}\right)^{-1}+\varepsilon^{N / 2}\right]
$$

such that

$$
\begin{aligned}
& \left\|\widetilde{\psi}_{\varepsilon, N}^{(1, m)}\right\|_{L_{2}(\Omega)}=1, \quad\left\|\widetilde{\psi}_{\varepsilon, N}^{(1, m)}-\frac{\mathrm{p}_{\varepsilon}^{1 / 2} \psi_{\varepsilon, N}^{(1, m)}}{\left\|\mathrm{p}_{\varepsilon}^{1 / 2} \psi_{\varepsilon, N}^{(1, m)}\right\|_{L_{2}(\Omega)}}\right\|_{L_{2}(\Omega)} \leqslant 2 \widehat{C}_{n}^{(1, m)} \delta \varepsilon^{N / 2-1}, \\
& \left\|\widetilde{\psi}_{\varepsilon, N}^{(1, m)}\right\| \mathrm{p}_{\varepsilon}^{1 / 2} \psi_{\varepsilon, N}^{(1, m)}\left\|_{L_{2}(\Omega)}-\mathrm{p}_{\varepsilon}^{1 / 2} \psi_{\varepsilon, N}^{(1, m)}\right\|_{L_{2}(\Omega)} \leqslant C_{N}^{(m)} \delta \varepsilon^{N / 2-1},
\end{aligned}
$$

where $C_{N}^{(m)}$ are positive constants independent of $\varepsilon$ and $\delta$. Here we have also used an obvious fact that $\left\|\mathrm{p}_{\varepsilon} \psi_{\varepsilon, N}^{(1, m)}\right\|_{L_{2}(\Omega)}$ can be estimated uniformly in $\varepsilon$ by a constant depending on $m$ and $N$.

Since $M$ is finite and fixed, and $m, p=1, \ldots, M$, by asymptotics (1.5) we conclude that there exists a constant $C$ independent of $\varepsilon, m, p=1, \ldots, M, m \neq p$, such that

$$
\left|\lambda^{(1, m)}(\varepsilon)-\lambda^{(1, p)}(\varepsilon)\right| \geqslant C, \quad m, p=1, \ldots, M, \quad m \neq p .
$$

Employing this estimate and choosing $\delta=\varepsilon^{2}$, we obtain the estimate

$$
\left|\frac{1}{1+\delta \lambda^{(1, m)}(\varepsilon)}-\frac{1}{1+\delta \lambda^{(1, p)}(\varepsilon)}\right|=\frac{\varepsilon^{2}\left|\lambda^{(1, m)}(\varepsilon)-\lambda^{(1, p)}(\varepsilon)\right|}{\left|1+\varepsilon^{2} \lambda^{(1, m)}(\varepsilon)\right|\left|1+\varepsilon^{2} \lambda^{(1, p)}(\varepsilon)\right|} \geqslant C \varepsilon^{2}
$$

for $\varepsilon$ small enough, $m, p=1, \ldots, M, m \neq p$, where $C$ is a positive constant independent of $\varepsilon, m$, and $p$. Hence, for $N \geqslant 5, m=1, \ldots, M$, and $\varepsilon$ small enough the intervals (4.3) contain no eigenvalues of $\mathcal{A}_{\varepsilon}$ except $\left(1+\varepsilon^{2} \lambda^{(1, m)}(\varepsilon)\right)^{-1}$. This eigenvalue is simple, since the corresponding eigenvalue $\lambda^{(1, m)}(\varepsilon)$ is simple. Thus, the linear combination $\widetilde{\psi}_{\varepsilon, N}^{(1, m)}$ is an orthonormalized in $L_{2}(\Omega)$ eigenfunction associated with $\left(1+\varepsilon^{2} \lambda^{(1, m)}(\varepsilon)\right)^{-1}$. Moreover, it is independent of $N$.

By Lemma 3.1 we have

$$
\left\|\mathrm{p}_{\varepsilon}^{1 / 2} \psi_{\varepsilon, N}^{(1, m)}\right\|_{L_{2}(\Omega)}=\sum_{j=0}^{N} c_{j}^{(m)} \varepsilon^{j}+\mathcal{O}\left(\varepsilon^{N+1}\right), \quad c_{j}^{(m)}=\text { const. }
$$

for all $N \geqslant 0, m=1, \ldots, M$. Hence, there exists a function $c_{m}=c_{m}(\varepsilon)$ such that

$$
c_{m}(\varepsilon)=\left\|\mathrm{p}_{\varepsilon}^{1 / 2} \psi_{\varepsilon, N}^{(1, m)}\right\|_{L_{2}(\Omega)}+\mathcal{O}\left(\varepsilon^{N+1}\right) \quad \text { for all } \quad N \geqslant 0
$$

The last equation, the identity $\delta=\varepsilon^{2}$, and (4.4) yield

$$
\left\|\widetilde{\psi}_{\varepsilon, *}^{(1, m)}-\mathrm{p}_{\varepsilon}^{1 / 2} \psi_{\varepsilon, N}^{(1, m)}\right\|_{L_{2}(\Omega)}=\mathcal{O}\left(\varepsilon^{N / 2+1}\right), \quad \widetilde{\psi}_{\varepsilon, *}^{(1, m)}:=c_{m}(\varepsilon) \widetilde{\psi}_{\varepsilon, N}^{(1, m)} .
$$

We use this equation and (3.52) and denote

$$
\psi^{(1, m)}(s, \xi, \varepsilon):=\mathrm{p}_{\varepsilon}^{-1 / 2}(s, \xi) \widetilde{\psi}_{\varepsilon, *}^{(1, m)}(s, \xi), \quad \Phi_{\varepsilon, N}^{(m)}(s, \xi):=\psi^{(1, m)}(s, \xi, \varepsilon)-\psi_{\varepsilon, N}^{(1, m)}(s, \xi),
$$

that yields

$$
\left\|\Phi_{\varepsilon, N}^{(m)}\right\|_{L_{2}(\Omega)}=\left\|\mathrm{p}_{\varepsilon}^{-1 / 2}\left(\widetilde{\psi}_{\varepsilon, *}^{(1, m)}-\mathrm{p}_{\varepsilon}^{1 / 2} \psi_{\varepsilon, N}^{(1, m)}\right)\right\|_{L_{2}(\Omega)}=\mathcal{O}\left(\varepsilon^{N / 2+1}\right) .
$$


It is also clear that the function $\psi^{(1, m)}(s, \xi, \varepsilon)$ solves $(2.7)$ with $\lambda(\varepsilon)=\lambda^{(1, m)}(\varepsilon)$. Hence, by $(3.47)$

$$
\widehat{\mathcal{H}}_{\varepsilon} \Phi_{\varepsilon, N}^{(m)}=\lambda^{(1, m)}(\varepsilon) \mathrm{p}_{\varepsilon} \Phi_{\varepsilon, N}^{(m)}+\widetilde{h}_{\varepsilon, N}^{(m)}, \quad \widetilde{h}_{\varepsilon, N}^{(m)}:=\left(\lambda^{(1, m)}(\varepsilon)-\lambda_{\varepsilon, N}^{(1, m)}\right) \mathrm{p}_{\varepsilon} \psi_{\varepsilon, N}^{(1, m)}-h_{\varepsilon, N}^{(1, m)}
$$

Due to this equation we can write the integral identity

$$
\left(\widehat{\mathcal{H}}_{\varepsilon} \Phi_{\varepsilon, N}^{(m)}, \Phi_{\varepsilon, N}^{(m)}\right)_{L_{2}(\Omega)}=\lambda^{(1, m)}(\varepsilon)\left(\mathrm{p}_{\varepsilon} \Phi_{\varepsilon, N}^{(m)}, \Phi_{\varepsilon, N}^{(m)}\right)_{L_{2}(\Omega)}+\left(\widetilde{h}_{\varepsilon, N}^{(m)}, \Phi_{\varepsilon, N}^{(m)}\right)_{L_{2}(\Omega)}
$$

From $(4.5),(3.46),(3.48),(1.5)$, and the smoothness of $\psi_{i}^{(n, m)}$ we derive

$$
\begin{aligned}
\left\|\widetilde{h}_{\varepsilon, N}^{(m)}\right\|_{C^{k}(\bar{\Omega})} & \leqslant\left|\lambda^{(1, m)}(\varepsilon)-\lambda_{\varepsilon, N}^{(1, m)}\right|\left\|\mathrm{p}_{\varepsilon} \psi_{\varepsilon, N}^{(1, m)}\right\|_{C^{k}(\bar{\Omega})}+\left\|h_{\varepsilon, N}^{(1, m)}\right\|_{C^{k}(\bar{\Omega})} \leqslant C \varepsilon^{N-1}\left(\left\|\mathrm{p}_{\varepsilon} \psi_{\varepsilon, N}^{(1, m)}\right\|_{C^{k}(\bar{\Omega})}+1\right) \\
& \leqslant C \varepsilon^{N-1}\left(\left\|\psi_{\varepsilon, N}^{(1, m)}\right\|_{C^{k}(\bar{\Omega})}+1\right)=\mathcal{O}\left(\varepsilon^{N-1}\right), \quad N \geqslant 5, \quad k \geqslant 0 .
\end{aligned}
$$

Together with $(1.5),(2.4),(2.5),(4.5),(4.7)$ it gives

$$
\begin{aligned}
\left\|\nabla_{(s, \xi)} \Phi_{\varepsilon, N}^{(m)}\right\|_{L_{2}(\Omega)}^{2} & \leqslant C\left(\mathrm{~A}^{(\varepsilon)} \nabla_{(s, \xi)} \Phi_{\varepsilon, N}^{(m)}, \nabla_{(s, \xi)} \Phi_{\varepsilon, N}^{(m)}\right)_{L_{2}(\Omega)} \\
& =C\left(\lambda^{(1, m)}(\varepsilon)\left(\mathrm{p}_{\varepsilon} \Phi_{\varepsilon, N}^{(m)}, \Phi_{\varepsilon, N}^{(m)}\right)_{L_{2}(\Omega)}+\left(\widetilde{h}_{\varepsilon, N}^{(m)}, \Phi_{\varepsilon, N}^{(m)}\right)_{L_{2}(\Omega)}\right)=\mathcal{O}\left(\varepsilon^{N}\right), \quad m=1, \ldots, M
\end{aligned}
$$

Combining (4.5) and (4.9), we conclude that asymptotics (1.7) hold true in $W_{2}^{1}(\Omega)$-norm.

We proceed to the proof of $(1.7)$ in $C^{k}\left(\Omega^{(t)}\right)$-norm. First we note that by the standard smoothness improving theorems we have $\psi_{\varepsilon}^{(1, m)} \in C^{\infty}\left(\overline{\Omega^{(t)}}\right)$ for all $t \in\left(0, s_{0} / 2\right)$. The rest of the proof follows from $(4.5),(4.6),(4.8),(4.9)$, the embedding of $W_{2}^{k+2}(\Omega)$ into $C^{k}(\bar{\Omega})$, and from the next lemma applied to $\Phi_{\varepsilon, N}^{(m)}$.

Lemma 4.1. Let $u_{\varepsilon}$ be a solution to the equation

$$
\widehat{\mathcal{H}}_{\varepsilon} u_{\varepsilon}=\lambda^{(1, m)}(\varepsilon) \mathrm{p}_{\varepsilon} u_{\varepsilon}+h, \quad h \in C^{\infty}(\bar{\Omega}) .
$$

Then $u_{\varepsilon} \in C^{\infty}\left(\overline{\Omega^{(t)}}\right)$ for all $t \in\left(0, s_{0} / 2\right)$, and

$$
\left\|u_{\varepsilon}\right\|_{W_{2}^{k}\left(\overline{\Omega^{(t)}}\right)} \leqslant C_{k} \varepsilon^{-2(k-1)}\left(\left\|u_{\varepsilon}\right\|_{W_{2}^{1}(\Omega)}+\|h\|_{W_{2}^{k-2}(\Omega)}\right)
$$

for all $k \geqslant 2, t \in\left(0, s_{0} / 2\right)$, where the constants $C_{k}$ are independent of $\varepsilon, h$ and $u_{\varepsilon}$.

Proof. The smoothness of $u_{\varepsilon}$ follows from that of $A_{i j}^{(\varepsilon)}, h, \mathrm{p}_{\varepsilon}$, and the smoothness improving theorems. For the sake of brevity we denote $\xi_{1}:=s, \xi:=\left(\xi_{1}, \xi_{2}, \xi_{3}\right)$.

Let $\chi_{1}^{(t)}=\chi_{1}^{(t)}\left(\xi_{1}\right)$ be an infinitely differentiable cut-off function equaling one as $\xi_{1} \in\left[t, s_{0}-t\right]$ and vanishing for $\xi_{1} \in[0, t / 2] \cup\left[s_{0}-t / 2, s_{0}\right]$. We fix $t \in\left(0, s_{0} / 2\right)$ and denote

$$
u_{\varepsilon}^{(t)}(\xi):=\chi_{1}^{(t)}\left(\xi_{1}\right) u_{\varepsilon}(\xi)
$$


It is straightforward to check that $u_{\varepsilon}^{(t)}$ solves the equation

$$
\begin{aligned}
& \widehat{\mathcal{H}}_{\varepsilon} u_{\varepsilon}^{(t)}=\lambda^{(1, m)}(\varepsilon) \mathrm{p}_{\varepsilon} u_{\varepsilon}^{(t)}+h \chi_{1}^{(t)}+\varepsilon^{-2} G_{1}^{(t)}\left(\varepsilon, \xi, u_{\varepsilon}, \frac{\partial u_{\varepsilon}}{\partial \xi_{i}}\right) \\
& G_{1}^{(t)}\left(\varepsilon, \xi, u_{\varepsilon}, \frac{\partial u_{\varepsilon}}{\partial \xi_{i}}\right)=\varepsilon^{2} \sum_{j=1}^{3} \frac{\mathrm{d} \chi_{1}^{(t)} \widetilde{A}_{i j}^{(\varepsilon)}}{\mathrm{d} \xi_{1}} \frac{\partial u}{\partial \xi_{j}}+\varepsilon^{2} u_{\varepsilon} \sum_{i=1}^{3} \frac{\partial}{\partial \xi_{i}} \widetilde{A}_{i j}^{(\varepsilon)} \frac{\mathrm{d} \chi_{1}^{(t)}}{\mathrm{d} \xi_{1}}
\end{aligned}
$$

We differentiate (4.11) with respect to $\xi_{1}$,

$$
\begin{aligned}
& \widehat{\mathcal{H}}_{\varepsilon} \frac{\partial u_{\varepsilon}^{(t)}}{\partial \xi_{1}}= \lambda^{(1, m)}(\varepsilon) \mathrm{p}_{\varepsilon} \frac{\partial u_{\varepsilon}^{(t)}}{\partial \xi_{1}}+\varepsilon^{-2} G_{2}^{(t)}\left(\varepsilon, \xi, u_{\varepsilon}^{(t)}, \frac{\partial u_{\varepsilon}^{(t)}}{\partial \xi_{i}}, \frac{\partial^{2} u_{\varepsilon}^{(t)}}{\partial \xi_{i} \partial \xi_{j}}\right) \\
&+\frac{\partial}{\partial \xi_{1}}\left(h \chi_{1}^{(t)}+\varepsilon^{-2} G_{1}^{(t)}\left(\varepsilon, \xi, u_{\varepsilon}, \frac{\partial u_{\varepsilon}}{\partial \xi_{i}}\right)\right), \\
& G_{2}^{(t)}\left(\varepsilon, \xi, u_{\varepsilon}^{(t)}, \frac{\partial u_{\varepsilon}^{(t)}}{\partial \xi_{i}}, \frac{\partial^{2} u_{\varepsilon}^{(t)}}{\partial \xi_{i} \partial \xi_{j}}\right)=\varepsilon^{2} \sum_{i, j=1}^{3} \frac{\partial}{\partial \xi_{i}} \frac{\partial \widetilde{A}_{i j}^{(\varepsilon)}}{\partial \xi_{1}} \frac{\partial u_{\varepsilon}^{(t)}}{\partial \xi_{j}}+\varepsilon^{2} \lambda_{1}^{(1, m)}(\varepsilon) \frac{\partial \mathrm{p}_{\varepsilon}}{\partial \xi_{1}} u_{\varepsilon}^{(t)} .
\end{aligned}
$$

Equation (4.12) implies the integral identity

$$
\begin{aligned}
\left(\mathrm{A}^{(\varepsilon)} \nabla \frac{\partial u_{\varepsilon}^{(t)}}{\partial \xi_{1}}, \nabla \frac{\partial u_{\varepsilon}^{(t)}}{\partial \xi_{1}}\right)_{L_{2}(\Omega)}= & \lambda^{(1, m)}(\varepsilon)\left(\mathrm{p}_{\varepsilon} \frac{\partial u_{\varepsilon}^{(t)}}{\partial \xi_{1}}, \frac{\partial u_{\varepsilon}^{(t)}}{\partial \xi_{1}}\right)_{L_{2}(\Omega)}+\varepsilon^{-2}\left(G_{2}^{(t)}, \frac{\partial u_{\varepsilon}^{(t)}}{\partial \xi_{1}}\right)_{L_{2}(\Omega)} \\
& -\left(h \chi_{1}^{(t)}+\varepsilon^{-2} G_{1}^{(t)}, \frac{\partial^{2} u_{\varepsilon}^{(t)}}{\partial \xi_{1}^{2}}\right)_{L_{2}(\Omega)}
\end{aligned}
$$

where we have by parts in the last term. We use (4.13) and integrate by parts in the second term in the right hand side of the last identity,

$$
\left(G_{2}^{(t)}, \frac{\partial u_{\varepsilon}^{(t)}}{\partial \xi_{1}}\right)_{L_{2}(\Omega)}=-\varepsilon^{2} \sum_{i, j=1}^{3}\left(\frac{\partial \widetilde{A}_{i j}^{(\varepsilon)}}{\partial \xi_{1}} \frac{\partial u_{\varepsilon}^{(t)}}{\partial \xi_{j}}, \frac{\partial^{2} u_{\varepsilon}^{(t)}}{\partial \xi_{i} \partial \xi_{1}}\right)_{L_{2}(\Omega)}+\varepsilon^{2} \lambda^{(1, m)}(\varepsilon)\left(\frac{\partial \mathrm{p}_{\varepsilon}}{\partial \xi_{1}} u_{\varepsilon}^{(t)}, \frac{\partial u_{\varepsilon}^{(t)}}{\partial \xi_{1}}\right)_{L_{2}(\Omega)} .
$$

We substitute this identity into (4.14) and proceed as in (4.9) employing Cauchy-Schwartz inequality, (1.5), and the explicit formulas for $G_{1}^{(t)}$ and $G_{2}^{(t)}$,

$$
\begin{aligned}
\left\|\nabla \frac{\partial u_{\varepsilon}^{(t)}}{\partial \xi_{1}}\right\|_{L_{2}(\Omega)}^{2} \leqslant & C\left(\mathrm{~A}^{(\varepsilon)} \nabla \frac{\partial u_{\varepsilon}^{(t)}}{\partial \xi_{1}}, \nabla \frac{\partial u_{\varepsilon}^{(t)}}{\partial \xi_{1}}\right)_{L_{2}(\Omega)} \\
\leqslant & C\left(\varepsilon^{-2}\left\|\frac{\partial u_{\varepsilon}^{(t)}}{\partial \xi_{1}}\right\|_{L_{2}(\Omega)}+\varepsilon^{-2} \sum_{i, j=1}^{3}\left\|\frac{\partial u_{\varepsilon}^{(t)}}{\partial \xi_{j}}\right\|_{L_{2}(\Omega)}\left\|\frac{\partial^{2} u_{\varepsilon}^{(t)}}{\partial \xi_{i} \partial \xi_{1}}\right\|_{L_{2}(\Omega)}\right. \\
& \left.\quad+\varepsilon^{-2}\left\|u_{\varepsilon}^{(t)}\right\|_{L_{2}(\Omega)}\left\|\frac{\partial u_{\varepsilon}^{(t)}}{\partial \xi_{1}}\right\|_{L_{2}(\Omega)}+\left(\|h\|_{L_{2}(\Omega)}+\varepsilon^{-2}\|u\|_{W_{2}^{1}(\Omega)}\right)\left\|\frac{\partial^{2} u_{\varepsilon}^{(t)}}{\partial \xi_{1}^{2}}\right\|_{L_{2}(\Omega)}\right) \\
\leqslant & C \varepsilon^{-4}\left(\|h\|_{L_{2}(\Omega)}+\|u\|_{W_{2}^{1}(\Omega)}\right)+\frac{1}{2}\left\|\nabla \frac{\partial u_{\varepsilon}^{(t)}}{\partial \xi_{1}}\right\|_{L_{2}(\Omega)}^{2} .
\end{aligned}
$$


Here and till the end of the proof by $C$ we indicate non-specific constants independent of $\varepsilon, u_{\varepsilon}$ and $h$. The obtained estimate implies

$$
\left\|\nabla \frac{\partial u_{\varepsilon}^{(t)}}{\partial \xi_{1}}\right\|_{L_{2}(\Omega)} \leqslant C \varepsilon^{-2}\left(\|h\|_{L_{2}(\Omega)}+\left\|u_{\varepsilon}\right\|_{W_{2}^{1}(\Omega)}\right) .
$$

Let us estimate $\left\|\nabla \frac{\partial u_{\varepsilon}^{(t)}}{\partial \xi_{i}}\right\|_{L_{2}(\Omega)}, i=2,3$. We could have tried to differentiate (4.11) with respect to $\xi_{i}$ and proceed as above. However, the function $\frac{\partial u_{\varepsilon}^{(t)}}{\partial \xi_{i}}$ does not vanish on $\left(0, s_{0}\right) \times \partial \omega$ and this is the main difficulty. This is why we have to employ a slightly different trick. We introduce an infinitely differentiable in $\bar{\omega}$ cut-off function $\chi_{2}=\chi_{2}(\xi)$ equaling one in a small neighborhood of $\partial \omega$ and vanishing outside a bigger neighborhood. Writing the equation for $u_{\varepsilon}^{(t)}\left(1-\chi_{2}\right)$ similar to (4.11) and proceeding as in (4.12), (4.15), one can show that

$$
\left\|\frac{\partial}{\partial \xi_{i}} u_{\varepsilon}^{(t)}\left(1-\chi_{2}\right)\right\|_{W_{2}^{1}(\Omega)} \leqslant C \varepsilon^{-2}\left(\|h\|_{L_{2}(\Omega)}+\left\|u_{\varepsilon}\right\|_{W_{2}^{1}(\Omega)}\right) .
$$

To estimate the same norm for $\chi_{2} u_{\varepsilon}^{(t)}$ we use the same approach as in the proof of Theorem 4 in [11], Chapter IV, Section 2.3.

In a small neighborhood of $\partial \omega$ we introduce new variables $\zeta=\left(\zeta_{1}, \zeta_{2}, \zeta_{3}\right)$, where $\zeta_{1}=\xi_{1}, \zeta_{2}$ is the arc length of $\partial \omega$, and $\zeta_{3}$ is the distance from the point to $\partial \omega$ measured in the direction of the inward normal. Then it follows from (4.11) that the function $v_{\varepsilon}^{(t)}=\chi_{2} u_{\varepsilon}^{(t)}$ satisfies the boundary value problem

$$
-\sum_{i, j=1}^{3} B_{i j}^{(\varepsilon)} \frac{\partial^{2} v_{\varepsilon}^{(t)}}{\partial \zeta_{i} \partial \zeta_{j}}=h \chi_{2} \chi_{1}^{(t)}+\varepsilon^{-2} \chi_{2} G_{1}^{(t)}+B_{0}^{(\varepsilon)} u_{\varepsilon}^{(t)}+\sum_{i=1}^{3} B_{i}^{(\varepsilon)} \frac{\partial u_{\varepsilon}^{(t)}}{\partial \zeta_{i}}, \quad \zeta \in\left(0, s_{0}\right) \times\left(0, \zeta_{2}^{(0)}\right) \times\left(0, \zeta_{3}^{(0)}\right),
$$

where $\zeta_{2}^{(0)}$ is the length of $\partial \omega, \zeta_{3}^{(0)}$ is a small fixed number. The operator in the left hand side of the last equation is elliptic uniformly in $\zeta$ and $\varepsilon$. The function $v_{\varepsilon}^{(t)}$ satisfies periodic boundary condition as $\zeta_{2}=0$ and $\zeta_{2}=\zeta_{2}^{(0)}$, and vanishes as $\zeta_{3}=0, \zeta_{3}=\zeta_{3}^{(0)}, \zeta_{1}=0, \zeta_{1}=s_{0}$. The coefficients $B_{i j}^{(\varepsilon)}, B_{i}^{(\varepsilon)}$ are infinitely differentiable and satisfy the estimates

$$
\left\|B_{i j}^{(\varepsilon)}\right\|_{C^{1}\left(\left[0, s_{0}\right] \times\left[0, \zeta_{2}^{(0)}\right] \times\left[0, \zeta_{3}^{(0)}\right]\right)} \leqslant C \varepsilon^{-2}, \quad\left\|B_{i}^{(\varepsilon)}\right\|_{C^{1}\left(\left[0, s_{0}\right] \times\left[0, \zeta_{2}^{(0)}\right] \times\left[0, \zeta_{3}^{(0)}\right]\right)} \leqslant C \varepsilon^{-2} .
$$

We differentiate (4.18) with respect to $\zeta_{2}$, and in the same fashion as in (4.12), (4.15), (4.16) we obtain

$$
\left\|\nabla \frac{\partial v_{\varepsilon}^{(t)}}{\partial \zeta_{2}}\right\|_{L_{2}\left(\left[0, s_{0}\right] \times\left[0, \zeta_{2}^{(0)}\right] \times\left[0, \zeta_{3}^{(0)}\right]\right)} \leqslant C \varepsilon^{-2}\left(\|h\|_{L_{2}(\Omega)}+\left\|u_{\varepsilon}\right\|_{W_{2}^{1}(\Omega)}\right) .
$$

Now we express the term $\frac{\partial^{2} v_{\varepsilon}^{(t)}}{\partial \zeta_{3}^{2}}$ from (4.18). Together with $(4.16),(4.17),(4.19)$ it gives the estimate

$$
\left\|\frac{\partial^{2} v_{\varepsilon}^{(t)}}{\partial \zeta_{3}^{2}}\right\|_{L_{2}\left(\left[0, s_{0}\right] \times\left[0, \zeta_{2}^{(0)}\right] \times\left[0, \zeta_{3}^{(0)}\right]\right)} \leqslant C \varepsilon^{-2}\left(\|h\|_{L_{2}(\Omega)}+\left\|u_{\varepsilon}\right\|_{W_{2}^{1}(\Omega)}\right) .
$$

This estimate and (4.16), (4.17), (4.19) imply (4.10) for $k=2$. To prove it for other $k$ 's, it is sufficient to proceed as above starting with differentiating the aforementioned equations for $\frac{\partial u_{\varepsilon}^{(t)}}{\partial \xi_{1}}, \frac{\partial}{\partial \zeta_{i}}\left(1-\chi_{2}\right) u_{\varepsilon}^{(t)}, \frac{\partial}{\partial \zeta_{i}} \chi_{2} u_{\varepsilon}^{(t)}$, $i=2,3$.

Acknowledgements. We thank D. Krejčiřík and the referees for valuable remarks which allowed us to improve the original version of the paper. A part of this work was done during the visit of D.B. to the Department of Engineering of University of Sannio, and he is grateful for the warm hospitality extended to him. 


\section{REFERENCES}

[1] N.S. Bakhvalov and G.P. Panasenko, Homogenization: Averaging processes in periodic media. Kluwer, Dordrecht/Boston/ London (1989).

[2] D. Borisov and P. Freitas, Singular asymptotic expansions for Dirichlet eigenvalues and eigenfunctions on thin planar domains. Ann. Inst. Henri Poincaré, Anal. Non Linéaire 26 (2009) 547-560.

[3] G. Bouchitté, M.L. Mascarenhas and L. Trabucho, On the curvature and torsion effects in one dimensional waveguides. ESAIM: COCV 13 (2007) 793-808.

[4] G. Cardone, T. Durante and S.A. Nazarov, The localization effect for eigenfunctions of the mixed boundary value problem in a thin cylinder with distorted ends. SIAM J. Math. Anal. (to appear).

[5] P. Duclos and P. Exner, Curvature-induced bound states in quantum waveguides in two and three dimensions. Rev. Math. Phys. 7 (1995) 73-102.

[6] P. Freitas and D. Krejčiř́ík, Location of the nodal set for thin curved tubes. Indiana Univ. Math. J. 57 (2008) 343-376.

[7] L. Friedlander and M. Solomyak, On the spectrum of the Dirichlet Laplacian in a narrow infinite strip, in Spectral theory of differential operators: M. Sh. Birman 80th anniversary collection, Adv. Math. Sci. 225, T. Suslina and D. Yafaev Eds., AMS Translations - Series 2, Providence (2008).

[8] L. Friedlander and M. Solomyak, On the spectrum of the Dirichlet Laplacian in a narrow strip. Israel J. Math. 170 (2009) 337-354.

[9] D. Grieser, Thin tubes in mathematical physics, global analysis and spectral geometry, in Analysis on Graphs and Its Applications, P. Exner, J.P. Keating, P. Kuchment, T. Sunada and A. Teplyaev Eds., Proc. Symp. Pure Math. 77, AMS, Providence (2008).

[10] D. Krejčiřík, Spectrum of the Laplacian in a narrow curved strip with combined Dirichlet and Neumann boundary conditions. ESAIM: COCV 15 (2009) 555-568.

[11] V.P. Mikhajlov, Partial differential equations. Moscow, Mir Publishers (1978).

[12] S.A. Nazarov, Dimension Reduction and Integral Estimates, Asymptotic Theory of Thin Plates and Rods 1. Novosibirsk, Nauchnaya Kniga (2001).

[13] O.A. Oleinik, A.S. Shamaev and G.A. Yosifyan, Mathematical problems in elasticity and homogenization, Studies in Mathematics and its Applications 26. Amsterdam etc., North-Holland (1992).

[14] G.P. Panasenko and M.E. Perez, Asymptotic partial decomposition of domain for spectral problems in rod structures. J. Math. Pures Appl. 87 (2007) 1-36.

[15] M.I. Vishik and L.A. Lyusternik, The asymptotic behaviour of solutions of linear differential equations with large or quickly changing coefficients and boundary conditions. Russ. Math. Surv. 15 (1960) 23-91. 